OPEN ACCESS

Edited by: Essaid Ait Barka,

Université de Reims Champagne Ardenne, France

Reviewed by:

Marc Ongena,

University of Liege, Belgium Jérôme Crouzet,

Université de Reims Champagne

Ardenne, France

*Correspondence:

Fouad Daayf

Department of Plant Science, 222,

Agriculture Building, University of

Manitoba, Winnipeg, MB, R3T 2N2,

Canada

Fouad.Daay@umanitoba.ca

${ }^{t}$ These authors have contributed equally to this work.

Specialty section: This article was submitted to Plant-Microbe Interaction, a section of the journal Frontiers in Plant Science

Received: 13 February 2015 Accepted: 29 May 2015 Published: 10 June 2015

Citation:

El Hadrami A, Islam MR, Adam LR and Daayf $F$ (2015) A cupin domain-containing protein with a quercetinase activity (NdQase) regulates Verticillium dahliae's pathogenicity and contributes to counteracting host defenses.

Front. Plant Sci. 6:440. doi: 10.3389/fpls.2015.00440

\section{A cupin domain-containing protein with a quercetinase activity (VdQase) regulates Verticillium dahliae's pathogenicity and contributes to counteracting host defenses}

\author{
Abdelbasset El Hadrami ${ }^{1,2+}$, Md. Rashidul Islam ${ }^{1,3+}$, Lorne R. Adam ${ }^{1}$ and Fouad Daayf ${ }^{1 \star}$ \\ ${ }^{1}$ Department of Plant Science, University of Manitoba, Winnipeg, MB, Canada, ${ }^{2}$ OMEX Agriculture Inc., Oak Bluff, MB, \\ Canada, ${ }^{3}$ Department of Plant Pathology, Bangladesh Agricultural University, Mymensingh, Bangladesh
}

We previously identified rutin as part of potato root responses to its pathogen Verticillium dahliae. Rutin was directly toxic to the pathogen at doses greater than $160 \mu \mathrm{M}$, a threshold below which many $V$. dahliae pathogenicity-related genes were up-regulated. We identified and characterized a cupin domain-containing protein (VdQase) with a dioxygenase activity and a potential role in $V$. dahliae-potato interactions. The pathogenicity of VdQase knock-out mutants generated through Agrobacterium tumefasciens-mediated transformation was significantly reduced on susceptible potato cultivar Kennebec compared to wild type isolates. Fluorescence microscopy revealed a higher accumulation of flavonols in the stems of infected potatoes and a higher concentration of rutin in the leaves in response to the VdQase mutants as compared to wild type isolates. This, along with the HPLC characterization of high residual and non-utilized quercetin in presence of the knockout mutants, indicates the involvement of VdQase in the catabolism of quercetin and possibly other flavonols in planta. Quantification of Salicylic and Jasmonic Acids (SA, JA) in response to the mutants vs. wild type isolates revealed involvement of VdQase in the interference with signaling, suggesting a role in pathogenicity. It is hypothesized that the by-product of dioxygenation 2-protocatechuoylphloroglucinolcarboxylic acid, after dissociating into phloroglucinol and protocatechuoyl moieties, becomes a starting point for benzoic acid and SA, thereby interfering with the JA pathway and affecting the interaction outcome. These events may be key factors for $V$. dahliae in countering potato defenses and becoming notorious in the rhizosphere.

Keywords: Verticillium dahliae Kleb., Verticillium wilt, biocontrol, plant extract, quercetinase, rutin, phloroglucinol, protocatechuoylphloroglucinol 


\section{Introduction}

Rutin, quercetin, kaempferol, and naringenin are secondary metabolites with a flavonol/none nucleus (i.e., rutin, quercetin, kaempferol, and naringenin; Supplementary Material 1) and often synthesized by higher plants in response to various abiotic and biotic stresses such as UV and microbial diseases, respectively (Harborne and Williams, 2000; Rozema et al., 2002; Grotewold, 2006; Daayf and Lattanzio, 2008). These naturallyoccurring anti-oxidants accumulate in various tissues and help prevent oxidative damage (Viornery et al., 2000; Andersen and Markham, 2006; Treutter, 2006). They can be toxic to the pathogen either directly or after oxidization into quinones, thus restricting its growth and development (Bennet and Wallsgrove, 1994; Barry et al., 2002; Treutter, 2006). The quercetin-3rutinoside aka rutin is a flavonol glycoside with a wide presence in fruits and vegetables. In legumes, rutin is released as a signal to initiate nodulation (Peters et al., 1986; Phillips, 1993). It is also released during the interaction with mycorrizhae and after wounding (Oka and Simpson, 1971; Poulin et al., 1993; Lagrange et al., 2001; Martin et al., 2001; Remy et al., 2009).

The co-evolution of pathogens with their hosts helped them tolerate such powerful anti-oxidants through degradation or detoxification. The bacteria colonizing the bowels and intestines of humans and animals are well known for their ability to degrade flavonols such as quercetin (Westlake et al., 1961; Winter et al., 1989, 1991; Kunst et al., 1997; Braune et al., 2001; Krogh et al., 2001; Rose and Fetzner, 2006). The phenomenon is less widespread among fungi and has been documented only in some ascomycetes (Aspergillus spp., Penicillium spp., Streptomyces spp., Pullularia sp., Fusarium oxysporum, Alternaria spp., Cephalosporium spp., and Diaporthe spp.) (Westlake et al., 1961; Bartz, 1971; Hund et al., 1999; Steiner et al., 2002; Merkens et al., 2007; Tranchimand et al., 2008) and a few basidiomycetes (Conidiophora puteana Karst, Lentinus lipedius Fr., Stereum sanguinolentum Fr., Armillaria mellea Karst, and Pleurotus ostreatus Kummer) (Sariaslani and Dalton, 1989). When Aspergillus flavus is grown on rutin (quercetin 3-O-rhamnoglucoside)-rich media as a singular carbon source, it activates glucosidases and esterase rutinases as well as an inducible quercetinase. The latter is a dioxygenase that is able to cleave two $\mathrm{C}$ - $\mathrm{C}$ bonds from the heterocyclic ring of quercetin, producing carbon monoxide and 2-protocatechuoylphloroglucinolcarboxylic acid (2-PCPGCA) (Child et al., 1963; Oka et al., 1971). Other fungi such as Pullularia spp. (Oka and Simpson, 1971), Fusarium spp. (Bartz, 1971), and

\footnotetext{
Abbreviations: $A_{367 \mathrm{~nm}}$, absorbance at $367 \mathrm{~nm}$; ATMT, Agrobacterium-mediated transformation; BA, benzoic acid; DMSO, dimethyl sulfoxide; DPH, 1,6diphenyl-1,3,5-hexatriene; DTT, Dithiothreitol; FW, fresh weight; IPM, Integrated Pest Management; IU, International unit of an enzyme; JA-pathway, Jasmonic Acid-pathway; LC-MS-MS, Liquid chromatography coupled to mass spectrophotometer; MAFFT, multiple sequence alignment based on fast Fourier transform; MES, 2-( $N$-morpholino)ethanesulfonic acid; MTT, methyl thiazolyl blue; ORF, open reading frame; PMS, phenazine methosulfate; PDA, Potato Dextrose Agar; 2-PCPGCA, 2-protocatechuoylphloroglucinolcarboxylic acid; PRRN, progressive pairwise alignment and iterative refinement; SA, salicylic acid; SDW, sterile distilled water; SD-DEPC-W, sterile distilled-DEPC-treated water; UPLC, Ultra Performance Liquid Chromatography.
}

other Aspergillus spp. (Oka et al., 1971) use the same degradation process in generating carbon monoxide while the majority of bacteria do not, due to the reduction of flavonols rather than their oxidation. Three prokaryotes possessing dioxygenases catalyzing the release of carbon monoxide analogously to fungal quercetinases are an exception (Wray and Abeles, 1993; El Hadrami et al., 2009).

Verticillium dahliae Kleb. is a soilborne pathogen that causes Verticillium wilt and threatens many important crops including potatoes (Daayf, 2015). This pathogen produces resting structures called microsclerotia that allow it to survive in the soil for several years but also to infect a wide range of hosts. Due to these two features, setting integrated management strategies to control this disease is challenging. In a previous study (Uppal et al., 2007, 2008), we reported on the effectiveness of selected biological treatments in reducing Verticillium wilt in potato plants grown either under controlled conditions or in the field. These included the use of bacterial isolates and extracts from Canada milkvetch. The mechanisms by which such disease protection took place were deemed to be through induced resistance (El Hadrami et al., 2011). Rutin was one of the main secondary metabolites that were induced, with a high accumulation in successful treatments, contrasting with lower induction in the non-effective ones.

The objectives of this study were to evaluate the effect of this differential activation of synthesis and/or accumulation of rutin on $V$. dahliae and to determine the mechanisms involved in the plant protection provided. Knowing the ability of fungi to pre- or post-transcriptionally interfere with host defenseresponses and signaling pathways (El Hadrami et al., 2009) and to detoxify accumulated secondary metabolites, we investigated the response of $V$. dahliae to the produced flavonoid. First, we examined in vitro the differential expression of selected $V$. dahliae genes activated in response to culture media amended with rutin and related flavonoids. Second, we isolated and characterized the Quercetinase (VdQase) involved in the dioxygenation of quercetin, the aglycone derived from rutin. Then, we generated a knockout mutant to further determine the role of VdQase in $V$. dahliae's pathogenesis and in countering potatoes defense responses.

\section{Materials and Methods}

\section{The Pathogen}

Two single spore- $V$. dahliae isolates namely Vd1396-9 (Vd9) and Vd1398-21 (Vd21) were selected for this study as highly aggressive isolates, in addition to their respective generated knockout mutants. The two wild-type isolates were the most aggressive among our collection regularly tested for pathogenicity on potato and/or sunflower (Uppal et al., 2007; Alkher et al., 2009). A weakly aggressive isolate Vs06-14 was also selected for pathogenicity comparison of the mutants. These isolates were grown either on solid culture media [i.e., Potato Dextrose Agar (PDA), water-agar] or on liquid media [i.e., Czapeck-Dox media, inorganic salt media, SDW, complete medium described by Dobinson et al. (1997)] depending on the objectives of each experiment. In addition to these isolates, other 
V. dahliae isolates were used in the in vitro and in planta tests for utilization of rutin and quercetin.

\section{Primers Design, Amplification, and Sequencing of Quercetinase-coding Sequences}

Several sets of primers were designed based on known quercetinases from Penicillium olsonii (Acc.: EU126643) Aspergillus japonicas (Acc.: Q7SIC2), and Streptomyces sp. strain FLA (Acc.: CAJ81053) to amplify putative quercetinasecoding sequences in $V$. dahliae. The primers pairs QueVd2F: 5'-CGCGTCTTGAGGTACTGGTT-3', QueVd2R: 5'-AGAGGC AGATCCGTGTGAGT-3'; QueVd3F: 5'-ACGACGGGCTCGTA ATACAC-3', QueVd3R: 5'-TCCGCTGGTAGCTCTTGTCT-3'; Q2FLAVd2F: 5'-ACACGGTGGTCATGCTTGTA-3', Q2FLAVd 2R: 5'-GGTGCCAGAAACGTCAAAGT-3' were retained for further investigations. Constitutive 18S RNA gene (Vd18SF: 5'-cggggaggtagtgacgataa-3'; Vd18SR: 5'-cattacggcggtcctagaaa$3^{\prime}$ ) was used as a control. The primer sets used to amplify the full length genes were FLAVd1F: 5'- CAAAGGACCTG ACGGAAGAC -3', FLAVd1R 5'- TCTCGCACATTCCAG ACTTG -3'; FLAVd2F: 5'-ATGACTCAAAAACAGACAGG-3', FLAVd2R 5'-CTAAGCCACAATCTCGTCAG-3', and Vd23F: 5'-ATGAGTGTTTCCGTTCACGC-3' and Vd23R1: 5'-CCA CTCTCCCCAGACTGAAG-3'. PCR assays were performed using either a $\mathrm{C} 1000^{\mathrm{TM}}$ or $\mathrm{MyCycler}{ }^{\mathrm{TM}}$ thermal cyclers (BIORAD, ON, Canada). The program consisted of $4 \mathrm{~min}$ initial denaturation at $94^{\circ} \mathrm{C}$ followed by 35 cycles with $30 \mathrm{~s}$ at $94^{\circ} \mathrm{C}$, $30 \mathrm{~s}$ at $57^{\circ} \mathrm{C}$ and $1 \mathrm{~min}$ at $72^{\circ} \mathrm{C}$ and a final extension at $72^{\circ} \mathrm{C}$ for $10 \mathrm{~min}$. The PCR products were visualized after amplification by electrophoresis on $1.5 \%$ agarose gel containing $1 \%$ ethidium bromide using a UV-transilluminator (AlphaImager, Alpha Innotech Co., Canada) equipped with a digital camera and a P93D digital monochrome printer (Mitsubishi Digital Electric America Inc.).

\section{RNA Isolation and Reverse-transcription of the Putative V. dahliae Quercetinase}

Total RNA was extracted using TRIzol ${ }^{\circledR}$ (Invitrogen Co.) according to the manufacturer's recommendations, out of $250 \mathrm{mg}$ FW of mycelia strains grown on liquid media control or amended with $1 \mathrm{ml}$ of commercial standard solutions of the tested compounds (rutin, quercetin, naringenin or phloroglucinol) calibrated at $100 \mu \mathrm{g} / \mathrm{ml}$ in dimethyl sulfoxide (DMSO). RNA quality was verified on $1.5 \%$ agarose gel and the concentrations and a Ultrospec 3100 spectrophotometer (Biochrom Ltd., Cambridge, UK) was used to estimate purity based on absorbance at $260 \mathrm{~nm}$ and the ratio A260/A280. The first-strand cDNA was synthesized using the M-MLV reverse transcriptase kit from Invitrogen Co. One microliter of total RNA was placed in a nuclease-free $200 \mu \mathrm{l}$ microcentrifuge PCR tube and mixed with $1 \mu \mathrm{l}$ of oligo $(\mathrm{dT})_{12-18}$ calibrated at $500 \mu \mathrm{g} \mathrm{ml}^{-1}$ and $1 \mu \mathrm{l}$ of $10 \mathrm{mM}$ dNTP mix and the volume was adjusted to $12 \mu \mathrm{l}$ using SD-DEPC-W. The mixture was then heated for $5 \mathrm{~min}$ at $65^{\circ} \mathrm{C}$ and quickly chilled on ice. After a brief centrifugation, the content was mixed with $4 \mu \mathrm{l}$ of $5 \mathrm{X}$ first-strand buffer, $2 \mu \mathrm{l}$ of $0.1 \mathrm{M}$ DTT and $1 \mu \mathrm{l}$ of $\mathrm{RNaseOUT}^{\mathrm{TM}}$ recombinant ribonuclease inhibitor calibrated at $40 \mathrm{U} \cdot \mu \mathrm{l}^{-1}$. The tubes were then gently mixed and incubated for $2 \mathrm{~min}$ at $37^{\circ} \mathrm{C}$ after which $1 \mu \mathrm{l} \mathrm{M-MLV} \mathrm{RT} \mathrm{(200} \mathrm{U)}$ was added to the contents and re-incubated for $50 \mathrm{~min}$ at $37^{\circ} \mathrm{C}$. The reaction was last inactivated by heating the mixture for $15 \mathrm{~min}$ at $70^{\circ} \mathrm{C}$. The synthesized first-strand CDNA was then used as a template for PCR with Q5F/R, QueVd2F/R, QueVd3F/R, Q2FLAVd2F/R primers as described above.

The PCR product amplified was purified using a MinElute ${ }^{\circledR}$ PCR purification Kit according to the manufacturer's instructions (Qiagen Inc.) and sent for sequencing (Macrogen Co., USA). Two independent sets of sequencing were performed out of two independent experiments.

\section{Sequences Analysis}

Products of sequencing were examined for any potential misinterpretation and submitted to a BLAST search for potential matches with stored sequences in the GeneBank. The cDNA sequences were also matched against other databases including Cogeme, MIT-Verticillium Genome project (www.broad.mit.edu/annotation/genome/verticillium_dahliae/), FASTA-UK, among others. The predicted proteins based on the best ORFs (NCBI-ORF) were also determined and matched for identities and similarities against most available sequences of quercetinases in the GenBank, EMBL, SwissProt, PIR, and ProDB as well as against known proteins from $V$. dahliae available in NCBI or MIT databases. ClustalW2 (Larkin et al., 2007) was used for DNA and protein sequences alignment to denote conservation, mutation, insertion/deletion events. Phylogenetic dendrograms were generated when necessary using Neighbor Joining algorithm based on percent identity calculated among sequences.

In silico analysis and annotation of the protein relied on the use of variety of tools to analyze the sequences (i.e., BLAST, NPS), predict the protein localization (i.e., PRED-CLASS, ProtFun), predict the presence of any putative signal peptide [i.e., SignalP (Bendtsen et al., 2004), SOSUlsignal (Fusetti et al., 2002)]. For topology prediction, secondary structure and generating the $3 \mathrm{D}$ structure of the protein the programs ProFunc (Laskowski et al., 2005), Phyre (Kelley and Sternberg, 2009) and WHATIF (Vriend, 1990) were used. The required pdb file required form some of the analysis were generated using either one of these program from the protein sequence or using 3D-JIGSAW (Bates et al., 2001). Superposition of the tested protein model with the reference dioxygenase template from $A$. japonicus or other templates was conducted using the programs SuperPose (Maiti et al., 2004) and WHATIF (Vriend, 1990).

\section{Assay of the Quercetinase Activity}

Two hundred mg of a freshly grown mycelium from isolates $\mathrm{Vd} 9$ and $\mathrm{Vd} 21$ were used for proteins extraction in $0.1 \mathrm{M}$ potassium phosphate buffer $\mathrm{pH}$ 7.0. The method described by Oka and Simpson $(1971)$ and Oka et al. $(1971,1972)$ was used to assess the quercetinase activity. This method follows the decrease in $A_{367 \mathrm{~nm}}$ as a result of quercetin breakdown $\left(\varepsilon=20,000 \mathrm{M} . \mathrm{cm}^{-1}\right)$ by a known volume of the enzyme added to a mixture consisting of $0.1 \mathrm{M}$ MES buffer at $\mathrm{pH} 6.0$, quercetin and copper. Ten to $20 \mu \mathrm{l}$ of protein extract were used for the enzymatic assays. One unit of enzyme activity (IU) was set to represent the amount of enzyme 
required to cleave $1 \mu \mathrm{M}$ of quercetin per hour under standard conditions. Every assay was repeated five times and the data was averaged after calculation of the activity.

$V$. dahliae quercetinase isozymes were separated by horizontal cellulose acetate gel electrophoresis on $76 \times 76 \mathrm{~mm}$ CA plates (Helena Laboratories, Beaumont, TX) in $25 \mathrm{mM}$ Tris-192 mM Glycine $\mathrm{pH} 8.5$ buffer. Fifty to $100 \mu \mathrm{l}$ of protein extract were used for the assays. Electrophoresis gels were run at $175-200 \mathrm{~V}$ for $15-20$ min till blue dye consisting of $0.25 \%$ bromophenol blue, $25 \%$ Ficoll reached the end of the gel. After a complete run, isozymes were stained in 0.1 M MES buffer, $\mathrm{pH} 6.0$ mixture containing $60 \mu \mathrm{l}$ of quercetin, $120 \mu \mathrm{l}$ of $\mathrm{CuSO}_{4}, 80 \mu \mathrm{l}$ of $10 \mathrm{mM}$ MTT, $80 \mu \mathrm{l}$ of $0.2 \%$ PMS blended with $1.6 \%$ agar. The cellulose acetate plates were then left in the staining solution at room temperature. When necessary, the gel was fixed in watermethanol-acetic acid (5:5:1, v/v/v) for $5 \mathrm{~min}$.

\section{Nucleic Acid Manipulations} Cloning and Targeted Disruption of $V$. dahliae VdQase A $3.8 \mathrm{~kb}$ VdQase coding sequence was amplified from the genomic DNA of Vd9 (Vd1396-9, a highly aggressive isolates of $V$. dahliae on potato using primers VdQase-FP ( $5^{\prime}$-AAGCTT AT GAGTGTTTCCGTTCACGC-3') and VdQase-RP (5'-AAGCTT TCATCCATTGCTGGCCTCGT- ${ }^{\prime}$ ) (underlined sequences indicate the HindIII restriction site in both primers) using the Phusion High Fidelity DNA polymerase (Invitrogen). After an initial denaturation at $98^{\circ} \mathrm{C}$ for $30 \mathrm{~s}$ the PCR conditions were denaturation at $98^{\circ} \mathrm{C}$ for $10 \mathrm{~s}$, annealing at $68^{\circ} \mathrm{C}$ for $45 \mathrm{~s}$ and elongation at $72^{\circ} \mathrm{C}$ for $2: 30 \mathrm{~min}$ for 35 cycles followed by a final extension at $72^{\circ} \mathrm{C}$ for $10 \mathrm{~min}$. The fragment was then cloned in pGEM T-Easy Vector (Promega) and confirmed by sequencing using M13 forward and reverse primers. The HindIII VdQase fragment was then sub-cloned at the same site of the binary plasmid pDHt [41] and confirmed by PCR, restriction digestion and sequencing. The cloned gene was then mutagenized using the EZ::TN system (Epicentre Technologies, Madison, WI) as described by Dobinson et al. (2004). The plasmids mutagenized was then electroporated into Escherichia coli strain DH10B (Invitrogen) and the EZ::TN insertion into the VdQase coding sequence were verified by PCR amplification using the plasmid from the chloramphenicol resistant clones as template with VdQase specific primers as mentioned above. The positive clones were then sequenced to determine the EZ::TN insertion sites in the VdQase coding region using the pMOD-2 forward (5'-GCCAACGACTACGCACTAGCCAAC-3') and reverse (5'-GAGCCAATATGCGAGAACACCCGAGAA- $3^{\prime}$ ) sequencing primers (Epicentre Technologies). The selected sequenced $\mathrm{pDHt}$ plasmid that contains the mutagenized VdQase gene was then transformed into Agrobacterium tumefaciens AGL-1 strains by electroporation using Gene Pulser (BioRad). The clones were then confirmed by PCR using the primers and conditions mentioned above.

\section{Production on VdQase Knockout Mutants}

Highly aggressive isolate $\mathrm{Vd} 9$ was selected as a wild type to generate the VdQase knockout mutant and conduct functional analyses. ATMT of $V$. dahliae was carried out using
Agrobacterium tumefasciens-mediated transformation (ATMT) as described by Dobinson et al. (2004) and Mullins et al. (2001) with slight modifications. $V$. dahliae wild type strain was grown on PDA for at least 1 week and conidia were harvested prior to co-cultivation. Timentin $(200 \mu \mathrm{g} / \mathrm{mL})$ was used instead of Moxalactum in the selection medium. Hygromycin Bresistant transformants were cultured in CM medium containing hygromycin in 24-well cell culture plates. Three to 5 days after, samples of each mutant culture were transferred onto PDA and single spore isolates were selected and re-cultured to produce mycelia and spores.

\section{Confirmation of Mutant Transformants}

The transformants were screened using PCR, southern blot hybridization and RT-PCR. PCR was used to confirm the gene-insertion mutants at the specific location of T-DNA integration using primers VdQase Upstream FP (5'-TGGTGT TGTTGCTCGGCATTTCGT- $3^{\prime}$ ), a primer that was designed few hundreds base pairs upstream of the start codon of VdQase, and the specific reverse primer for VdQase gene mentioned above. For southern hybridization, genomic DNA was extracted from the wild type and the mutant isolates. The probe hygromycin B was amplified from the pSK846 using primers HyF (5' -TCAGCTTCGATGTAGGAGGG-3') and HyR (5'-TTCTACACAGCCATCGGTCC-3') and was labeled according to the GE Healthcare instruction manual. For blotting, genomic DNA $(20 \mu \mathrm{g})$ of both the wild type and mutant transformants was digested with EcoRI (Promega) and separated on agarose gel before being transferred onto Amersham Hybond $\mathrm{N}^{+}$membranes (GE Healthcare). The hybridizations and signals detection on the Kodak X-Omat film as described in the Alkhos labeling kit (GE Health Care). For RT-PCR, total RNA was extracted from the wild type and the transformants, grown on CDX and CM media, respectively, using TRIzol reagent (Invitrogen). c-DNA was synthesized as described above using the primer pair VdQase RT FP (5'-GGCAAGGAC TGGAGGAGTTGATT- $\left.3^{\prime}\right)$ and VdQase RT RP ( $5^{\prime}$-GTGATTGC CATT GCCGACGGTA-3). PCR conditions were $95^{\circ} \mathrm{C}$ for $3 \mathrm{~min}$ initial denaturation followed by 35 cycles of denaturation at $95^{\circ} \mathrm{C}$ for $30 \mathrm{~s}$, annealing at $60^{\circ} \mathrm{C}$ for $30 \mathrm{~s}$, and extension at $72^{\circ} \mathrm{C}$ for $1 \mathrm{~min}$ and a final extension $72^{\circ} \mathrm{C}$ for $5 \mathrm{~min}$.

\section{Pathogenicity Testing}

The single spore $V$. dahliae wild type and mutant isolates were maintained on PDA at $20 \pm 2{ }^{\circ} \mathrm{C}$ until used. Pathogenicity tests were conducted in triplicates as described by Alkher et al. (2009) and the whole experiment was repeated three times. Briefly, Fourweek-old potato plants were used for inoculations. Inoculum of each $V$. dahliae isolates were prepared from single-spore cultures grown on PDA for 2 weeks at $20^{\circ} \mathrm{C}$ (Fisher Scientific Incubator, Model 146E). Conidial suspensions were prepared to a final concentration of $1 \times 10^{6}$ conidia/mL. The plants were inoculated via "root dip" inoculation (Alkher et al., 2009). Briefly, the soil was gently washed from the uprooted potato plant roots with water followed by trimming the root tips with scissors. The root systems were then immersed in the conidial suspensions for $1 \mathrm{~min}$ before being transplanted. The plants for the wounded 
control treatment had their root tips cut before immersion in sterile distilled water (SDW). The inoculation experiments were conducted with three replications. In Arabidopsis thaliana, the inoculation was conducted with slight modifications.

\section{In Vitro and In Planta Utilization of Rutin and Quercetin}

Rutin and quercetin in vitro utilization by the wild type and VdQase mutants was assessed as previously described by El Hadrami et al. (2011) with a slight modification. Quantification was conducted using HPLC and the data was expressed as percentage of the untreated control for the in vitro tests. All analyses were conducted in triplicates and repeated at least twice. The selected isolates (Vd9, Vd9+25-5, Vd9+25-7, and Vs06-14) were grown in $100 \mathrm{ml}$ of Czapeck-Dox (CDX) liquid medium (Hurst et al., 2002), either non-amended, or amended with $1 \mathrm{ml}$ of commercial standard solutions of the tested compounds calibrated at $100 \mu \mathrm{g} / \mathrm{ml}$ in dimethyl sulfoxide (DMSO). Media amended only with $1 \mathrm{ml}$ of DMSO were used as controls. The inoculated media as well as the controls were kept at room temperature on a shaker (Edison, NJ, USA) in the dark at $120 \mathrm{rpm}$. Samples consisting of $1-\mathrm{ml}$ solutions were taken at 0 , $3,5,8,15,21$, and 28 days after transfer. They were immediately analyzed by HPLC or kept at $-20^{\circ} \mathrm{C}$ until needed. Identification of each compound or its product of degradation was based on characteristics including absorption spectra, retention times, comparison and co-elution with commercial standards and on TLC and HPLC. Quantitative analyses were performed by HPLC using the peak area with reference to a standard curve made for each commercial standard (Sigma-Aldrich Co.). Three replicates per treatment $\mathrm{x}$ isolate combination were considered for this experiment. Quantifications of the added compounds and their products of degradation were performed in triplicate and the data are reported as the recorded average values.

\section{Determination of SA and JA Levels}

Leaf samples were collected from potato plants inoculated with wild type and mutant isolates at 2, 3 and 4 w.p.i. for the determination of SA and JA. For SA, $500 \mathrm{mg}$ of tissues were reduced to powder under liquid nitrogen and suspended in $500 \mu \mathrm{l}$ of $80 \%$ methanol amended with $10 \mu \mathrm{l}$ of $\mathrm{O}$-anisic acid (Sigma-Aldrich) calibrated at $250 \mathrm{ng} / \mathrm{ml}$ as an internal standard (Meuwly and Métraux, 1993). The samples were transferred overnight onto a shaker placed at $4{ }^{\circ} \mathrm{C}$ then centrifuged at 7000 $g$ for $5 \mathrm{~min}$. After evaporation of the methanol fraction, free SA was extracted using ethyl acetate (v/v) and re-suspended in pure methanol. For the extraction of bound SA, the aqueous phases were hydrolyzed with $4 \mathrm{~N} \mathrm{HCl}$ at $100^{\circ} \mathrm{C}$ for $2 \mathrm{~h}$ followed by two times extraction with ethyl acetate. After evaporation of the organic phases, the residues were re-suspended in pure methanol. Samples were run on a Waters Alliance 2695 HPLC equipped with at Waters 996 PDA and W2475 Fluorescence detectors. Samples were injected onto an Agilent Poroshell 120 EC-C18 column $(4.6 \times 100 \mathrm{~mm}, 2.7 \mu \mathrm{m})$ with mobile phase A (water with $0.1 \% O$-phosphoric acid) and $\mathrm{B}$ (Acetonitrile) at a flow rate of $1 \mathrm{ml} / \mathrm{min}$. The following gradient was used for the elution:
Time (min) - \%A - \%B 0-100-0; 2-95-5; 5.6-90-10; 8-80-20; 12 $65-35 ; 17.2-50-50 ; 19.2-25-75 ; 22-0-100 ; 24.8-100-0 ; 26-100-0$. The W2475 fluorescence detector was set for $290 \mathrm{~nm}$ excitation wavelength and an emission scanning from 300 to $500 \mathrm{~nm}$. SA was detected at $390 \mathrm{~nm}$ while the $O$-anisic acid internal standard was measured at $350 \mathrm{~nm}$. Molar concentration of SA was adjusted by expected molar concentration of the internal standard.

JA was quantified using $( \pm)$-9,10-dihydrojasmonic acid DHJA (OlChemIm Ltd. Czech Republic) as an internal standard. For the extraction, $500 \mathrm{mg}$ of tissues were reduced to powder using liquid nitrogen and suspended in $5 \mathrm{ml}$ of extraction solvent (HCl:2-propanol: $\mathrm{H}_{2} \mathrm{O}$ in a ratio of 2:1:0.002) containing $50 \mu \mathrm{l}$ of DHJA calibrated at $1 \mu \mathrm{g} / \mathrm{ml}$. The mixtures were shaken for $30 \mathrm{~min}$ at $4^{\circ} \mathrm{C}$ then mixed with $7 \mathrm{ml}$ of dichloromethane. After centrifugation at $7000 \mathrm{~g}$ for $5 \mathrm{~min}$, the supernatants were evaporated to dryness and re-suspended in 50\% methanol. Samples were injected onto an Acquity UPLC equipped with a Micromass Quattro micro LC/MS/MS in ESI mode. Samples were injected onto a Phenomenex Kinetex $(100 \times 2.1 \mathrm{~mm} 1.7 \mu \mathrm{m})$ column with mobile phase A (water containing $0.1 \%$ formic acid) and $\mathrm{B}$ (methanol containing $0.1 \%$ formic acid) at $0.22 \mathrm{ml} / \mathrm{min}$ with the following gradient: Time (min)-\%A-\%B: 0-70-30; 2-70$30 ; 12-0-100 ; 13-0-100 ; 15-70-30$. JA and DHJA were quantified as a peak area of MRM data.

\section{Flavonoid Detection by Fluorescence Microscopy}

In situ detection of flavonoids, including flavonols, was done using fluorescence microscopy. Stem samples were taken $28 \mathrm{dpi}$ from the bottom, middle and top parts of the tested potato plants. Free hand cross-sections were immersed in NEU reagent (1\% methanolic 2-aminoethyl diphenylborinate, Sigma-Aldrich D9754) before observation under a fluorescence microscope (Vanguard Microscopes, Model 1486FL, VEE GEE Scientific Inc., Kirkland, WA USA) with filter set Exciter, Dichroic and Emitter wavelengths of 365,400 , and $535 \mathrm{~nm}$, respectively. The NEU reagent reveals the presence of many flavonoids in bright yellow or orange under UV-366 nm.

\section{Experimental Design and Data Analysis}

All experimental trials were done in triplicates and repeated at least once over time unless otherwise stated. Quantitative data was submitted to ANOVA analysis using Statistica software (Statsoft Inc., 1999). The pairwise comparison of the means was performed according to using Newman-Keuls test at $P<$ 0.05 . Quercetinase activity was based on five independent assays performed for each treatment. To determine the enzymatic activity data points were collected and used in a linear regression of absorbance decrease at $367 \mathrm{~nm}\left(A_{367 \mathrm{~nm}}\right)$ over time.

Sequences alignment was conducted using ClustalW2 (Larkin et al., 2007) and dendrograms were generated using algorithms such as PRRN (progressive pairwise alignment and iterative refinement; Gotoh, 1999) and multiple sequence alignment based on fast Fourier transform, MAFFT (Katoh et al., 2002). Neighbor-Joining algorithm was used to generate phylogenetic dengdrograms based on the percent identity among the compared sequences. 
Other programs used during the in silico characterization of the protein generated specific statistical parameters to assess the robustness of the predictions.

\section{Results}

\section{Gene Expression in V. dahliae Grown in Flavonol-amended Liquid Media}

Primer sets QueVd2F/R, QueVd3F/R, Q2FLAVd1F/R, and $\mathrm{Q} 2 \mathrm{FLAVd} 2 \mathrm{~F} / \mathrm{R}$ were designed based on known quercetinases from $P$. olsonii, Aspergillus japonicus and Streptomyces sp. strain FLA, respectively, to amplify putative quercetinase-coding sequences in $V$. dahliae. RT-PCR products amplified with these primer sets (Figure 1A) were sequenced and BLAST-searched (Altschul et al., 1997) against the annotated $V$. dahliae genome available on MIT website (www.broadmit.edu/). The analysis showed $85-100 \%$ identity of the sequences with cupin $1, \mathrm{Zn}$ finger $\mathrm{C} 2 \mathrm{H} 2$ transcripts fully sequenced and annotated with no assigned function, and located on chromosome 3 of the two reference isolates in the database (Table 1). Using the original quercetinase sequences from $P$. olsonii and $A$. japonicus in the BLAST-search revealed $82 \%$ identity with the same cupin domain-containing protein matching the sequences amplified with QueVd2F/R and QueVd3F/R. Against V. dahliae VdLs.17 ORF VDAG_02532.1 the parameters of the match were: Score = 87.81 bits (216); Expect $=8.88257 \mathrm{e}^{-18}$; Identities $=82 / 342$ $(23 \%)$; Positives $=143 / 342(41 \%)$. The ORF was then named VdQase.

BLAST-search of the sequenced transcripts amplified using primer sets QueVd2F/R, QueVd3F/R against other databases revealed homologies with conserved transcription and DNA binding factors that are often involved in metal binding (i.e., copper; Acc. emb|CAP79125.1) or in the protection against organic peroxides (i.e., AhpA protein; Acc. gb|AAT02761.1) (Table 2). Transcripts amplified using primer set Q2FLAVd2F/R showed similarities with membrane permeases, sugar transporters/permeases, and structural and transmembrane proteins (Table 2). Interestingly, known quercetinases from $P$. olsonii and A. japonicus showed similarities with these same proteins.

The percent identity of the amplified DNA sequences from both isolates $\mathrm{Vd} 9$ and $\mathrm{Vd} 21$ with well characterized quercetinases from P. olsonii and Streptomyces sp. strain FLA averaged 50\% (Table 3).

The incubation of the tested $V$. dahliae isolates (Vd9 and $\mathrm{Vd} 21$ ) in presence of rutin, quercetin and naringenin induced a substantial accumulation of VdQase transcripts as compared to the untreated control or in mycelia incubated in presence of phloroglucinol (Figure 1B). This allowed us to amplify homolog genes' full-length sequences of $\sim 1.5$ and $2.2 \mathrm{~Kb}$ with Vd23F/R1 and FLAVd2F/R1 primer pairs, respectively. No effect of the treatments was recorded on the expression of the $18 \mathrm{~S}$ rRNA used as a reference gene (Figure 1C). The analysis of these transcripts using Phyre (Kelley and Sternberg, 2009) and ConFunc (Wass and Sternberg, 2008) revealed a strong homology and 24-26\% identity with RmlC-like Cupin from $A$. japonicus (SCOP Code: d1juha_) or Bacillus subtilis (SCOP Code: d1y3ta1); both known as Quercetin 2,3-dioxygenase-like proteins (Supplementary Material Xls file 1).

\section{In Silico Annotation, Structure Identification and Functional Analysis of the First Quercetinase in $V$. dahliae (VdQase)}

The predicted protein from transcript homolog of VDAG_02532.1 (www.broadmit.edu/) had 1047 residues. No signal peptide was predicted using either SignalP (Bendtsen et al., 2004) or SOSUIsignal (Gomi et al., 2004) programs. Residues comprised between 2 and 323 were revealed to be encoded by the quercetinase gene using ProFunc program (Laskowski et al., 2005). Using SCRATCH program (Cheng et al., 2005), three domains were predicted. Domain 1 spans from residue 1 to 559; domain 2 ranges from 560 to 769 and the third domain contains residues 770-1047. Sixteen cysteines were detected and six disulfide bonds were predicted (Cys1-Cys2: 340-361; 220-252; 824-854; 625-628; 88-995; 654-686). Annotation using various programs such as SMART, SWISS-Model, TMHMM2 and PROSCAN (Gattiker et al., 2002; Katoh et al., 2002; Rozema et al., 2002; Schwede et al., 2003; Letunic et al., 2004; Larkin et al., 2007) allowed for the prediction with confidence of several conserved motifs including a Cupin 2, a homeodomain (HOX) and a $\mathrm{ZnF}$ _C2H2 as well as a fungal-specific transcription factor (Figure 2).

Using 3D-JIGSAW (Bates et al., 2001), a pdb signature was generated for the characterized protein. The analysis of this signature using the program ProFunc (Laskowski et al., 2005) allowed for the characterization of the topology and the secondary structure of the protein (Supplementary Material $2,3)$. The PROMOTIF module of this program showed that the functional protein contains 316 residues among which 114 (36.1\% of the residues) form strands, 15 (4.7\%) alpha-helices. The remaining 187 (59.2\%) form either $\beta$-hairpins, $\alpha$ - or $\beta$ sheets and bulges or one $\gamma$-turn. A predicted 3D-model of the protein was also established from this analysis (Supplementary Material 4). The same program generates also a PROCHECK analysis of the protein. The Ramachandran plot (Ramachandran et al., 1963) revealed a significant $84.6 \%$ of residues in most favored regions within the phipsi core $[\mathrm{A}, \mathrm{B}, \mathrm{L}]$ (Supplementary Material 5). Additional analysis included in the ProFunc reports such as InterPro scan (Quevillon et al., 2005) of the sequence motifs and the search of the sequence against superfamily HMM library (Finn et al., 2006) confirmed once again the presence of a Cupin_2 motif and the belonging to the RmlC-like cupin superfamily (Supplementary Material 6).

Sequence matching against existing PDB entries revealed certainty matches (E-value $<1.00 e-06)$ with quercetin 2,3dioxygenases from $A$. japonicus and B. subtilis. BLAST search against Uniprot database showed also certainty matches ( $E$ value $<1.00 e-06$ ) with dioxygenases from A. fimugatus, A. flavus, and proteins from P. chrysogenum and Neosartorya fisheri, both belonging to the cupin superfamily. In terms of ligand-binding templates, the analysis showed 12 significant hits, two of which were probable $(1.00 e-06<$ E-value $<0.01)$ with "TLAc0047" from B. subtilis and "_CUc0024" from A. japonicas, and one 


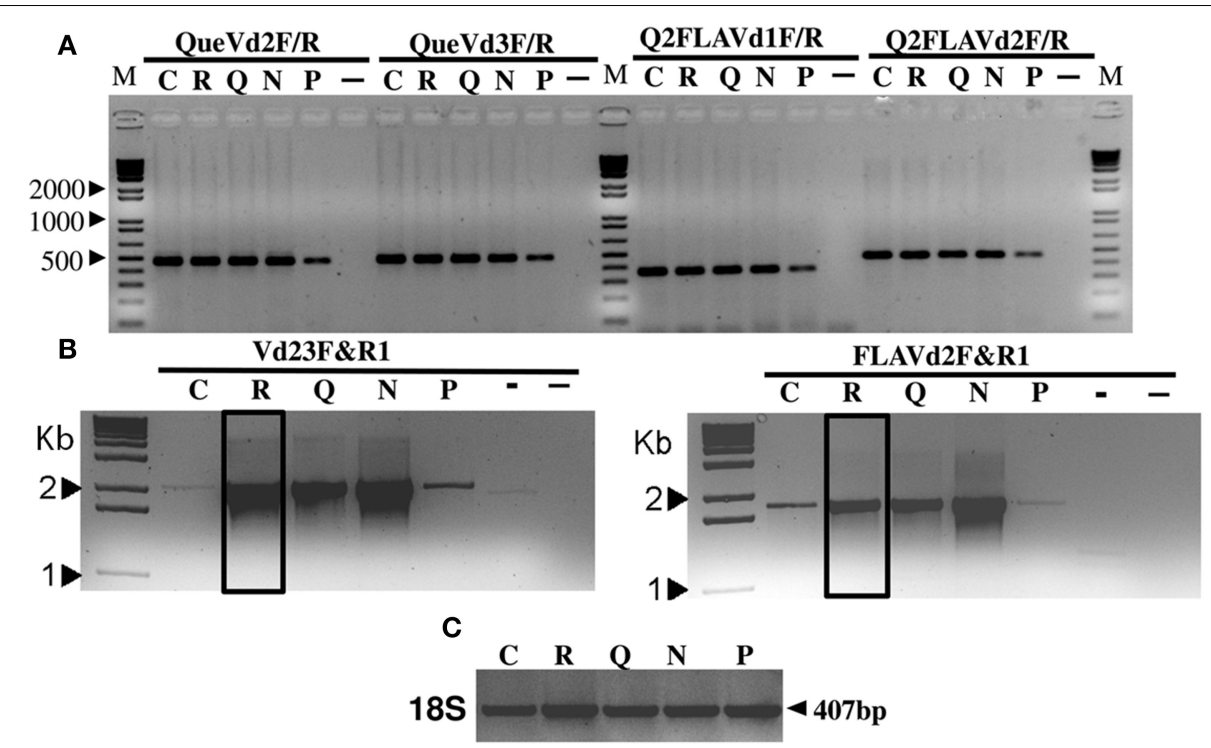

FIGURE 1 | RT-PCR products amplified with primer pairs QueVd2F/R, QueVd3F/R, Q2FLAVd1F/R, and Q2FLAVd2F/R out of mRNA isolated from $V$. dahliae isolate $V d 9$ grown on liquid media control $(C)$ or amended with rutin $(R)$, quercetin $(Q)$, naringenin $(N)$, or phloroglucinol (P). (-): negative control. (-): double negative control using plant RNA. Similar profiles were detected using isolate Vd21 and were not included for space convenience. (A) represents the initial amplification of the transcripts with various sets of primers while (B) represents the relative abundance of the full length transcripts of each putative VdQase gene amplified using Vd23F/R1 and FLAVd2F/R1 primer sets. (C) represents the amplification of $18 \mathrm{~S}$ rRNA used as reference gene.

TABLE 1 | Matches of the amplified V. dahliae sequences against annotated V. dahliae genome available through MIT website (www.broadmit.edu/).

\begin{tabular}{|c|c|c|c|c|}
\hline Primers set and target & Score (Bits) & Expect & Identities (\%) & Positives (\%) \\
\hline \multicolumn{5}{|l|}{ QueVd2 F/R } \\
\hline V. dahliae VdLs.17: VDAG_02532.1: cupin domain-containing protein & $263.462(672)$ & 0.0 & $127 / 127$ (100\%) & $127 / 127(100 \%)$ \\
\hline V. albo-atrum VaMs.102: VDBG_02075.1: conserved hypothetical protein & $227.254(578)$ & 0.0 & $112 / 127$ (88\%) & $114 / 127$ (89\%) \\
\hline \multicolumn{5}{|l|}{ QueVd3 F/R } \\
\hline V. dahliae VdLs.17: VDAG_02532.1: cupin domain-containing protein & $288.5(737)$ & 0.0 & $144 / 148$ (97\%) & $145 / 148(97 \%)$ \\
\hline V. albo-atrum VaMs.102: VDBG_02075.1: conserved hypothetical protein & $239.58(610)$ & 0.0 & $122 / 147$ (82\%) & $130 / 147$ (88\%) \\
\hline \multicolumn{5}{|l|}{ Q2FLAVd2 F/R } \\
\hline V. dahliae VdLs.17: VDAG_02534.1: conserved hypothetical protein & $172.17(435)$ & $3.9 e^{-44}$ & $103 / 103(100 \%)$ & $103 / 103(100 \%)$ \\
\hline V. albo-atrum VaMs.102: VDBG_02078.1: conserved hypothetical protein & $93.2041(230)$ & $2.3 e^{-20}$ & $60 / 104(57 \%)$ & $69 / 104(66 \%)$ \\
\hline
\end{tabular}

possible match $(0.01<$ E-value $<0.1)$ with “_CUc0075” form A. japonicus. The reverse template comparison $v$ s. the structures in PDB revealed a certainty match $(E$-value $<1.00 e-06)$ with "pdb_1juh", which is the quercetin 2,3-dioxygenases from $A$. japonicus (Supplementary Material Xls file 1).

Further analysis using WhatIf (Vriend, 1990) and SuperPose (Maiti et al., 2004) programs allowed for the superposition of the $3 \mathrm{D}$-predicted structure of the protein with the certainty template match "pdb_1juh." This along with the generated ClustalW analysis of the aligned sequences from the template revealed a high degree of conservation as well as conserved and semiconserved substitution in the amino-acid sequences (Figure 3).

\section{In Vitro Assay of the Enzymatic Activity}

The examination of quercetinase in vitro (Figure 4A) revealed an activity of $160 \mathrm{IU}$; one IU being $1 \mu$ mole of quercetin cleaved per hour under standard conditions, with a $\mathrm{Km}$ ranging from $0.0025-0.0036$ for quercetin (calculated in $\mu$ mol quercetin $\min ^{-1}$ ) and $0.06-0.08$ for $\mathrm{O}_{2}$ (estimated). The specific activity average was $2516 \mu \mathrm{mol}$ quercetin $\mathrm{min}^{-1} \mathrm{mg}^{-1}$ protein $\mathrm{g}^{-1}$ mycelium FW. The use of dioxygenase inhibitors such as kojic acid or prohexadione-Ca abolished the quercetinase activity (El Hadrami et al., 2011). Similarly, using chelated copper (Cu-EDTA) did not yield any enzymatic activity (data not shown).

The separation of $V$. dahliae quercetinase isozymes on horizontal cellulose acetate gel electrophoresis showed at least three isoforms of the enzyme when the proteins were extracted from mycelia grown on liquid media amended with rutin, quercetin or naringenin. When the mycelia were collected from the control or from media amended with phloroglucinol, only one isoform was detected (Figure 4B). The overall activity of the 
TABLE 2 | Matches of the amplified $\boldsymbol{V}$. dahliae sequences against NCBI GenBank.

\begin{tabular}{|c|c|c|c|c|}
\hline $\begin{array}{l}\text { Matching } \\
\text { Accession }\end{array}$ & Putative function & Organism & Score (Bits) & $E$ value \\
\hline \multicolumn{5}{|l|}{ Vd21Q1-QUEVd2F/R } \\
\hline ref|XP_389742.1| & hypothetical protein FG09566.1 & Gibberella zeae $\mathrm{PH}-1$ & 100 & $6 e-20$ \\
\hline ref|XP_389223.1] & hypothetical protein FG09047.1 & Gibberella zeae $\mathrm{PH}-1$ & 99.8 & $7 e-20$ \\
\hline ref|XP_001909254.1| & unnamed protein product & Podospora anserina & 99.4 & $1 e-19$ \\
\hline ref|XP_389219.1| & hypothetical protein FG09043.1 & Gibberella zeae PH-1 & 99.0 & $1 e-19$ \\
\hline ref|XP_001933004.1| & conserved hypothetical protein conserved & Pyrenophora tritici-repentis Pt-1C-BFP & 96.7 & $6 e-19$ \\
\hline ref|XP_001932443.1| & hypothetical protein & Pyrenophora tritici-repentis Pt-1C-BFP & 96.3 & $8 e-19$ \\
\hline ref|XP_001596875.1| & hypothetical protein SS1G_03098 & Sclerotinia sclerotiorum 1980 & 95.5 & $1 e-18$ \\
\hline gb|EDP56789.1| & homeobox $\mathrm{C} 2 \mathrm{H} 2$ transcription factor, putative & Aspergillus fumigatus A1163 & 95.1 & $2 e-18$ \\
\hline ref|XP 752922.1 & homeobox and $\mathrm{C} 2 \mathrm{H} 2$ transcription factor & Aspergillus fumigatus Af293 & 95.1 & $2 e-18$ \\
\hline ref|XP_001223987.1| & hypothetical protein CHGG_04773 & Chaetomium globosum CBS 148.51 & 94.7 & $2 e-18$ \\
\hline ref|XP_001796737.1| & hypothetical protein SNOG_06363 & Phaeosphaeria nodorum SN15 & 94.4 & $3 e-18$ \\
\hline ref|XP_001555211.1| & hypothetical protein BC1G_06341 & Botryotinia fuckeliana B05.10 & 93.6 & $5 e-18$ \\
\hline ref|XP_001264194.1| & homeobox $\mathrm{C} 2 \mathrm{H} 2$ transcription factor, putative & Neosartorya fischeri NRRL 181 & 93.2 & $7 e-18$ \\
\hline emb|CAP79125.1| & Pc06g01320, similar to copper homeostasis & Penicillium chrysogenum Wisconsin 54-1255 & 78.2 & $2 e-13$ \\
\hline emb|CAP97951.1| & Pc22g06630, DNA binding domain & Penicillium chrysogenum Wisconsin 54-1255 & 77.8 & $3 e-13$ \\
\hline$\overline{\text { ref|XP_682597.1 }}$ & hypothetical protein AN9328.2 & Aspergillus nidulans FGSC A4 & 77.0 & $5 e-13$ \\
\hline ref|XP_388085.1| & hypothetical protein FG07909.1 & Gibberella zeae $\mathrm{PH}-1$ & 75.1 & $2 e-12$ \\
\hline ref|XP_001273098.1| & homeobox $\mathrm{C} 2 \mathrm{H} 2$ transcription factor, putative & Aspergillus clavatus NRRL 1 & 74.7 & $3 e-12$ \\
\hline gb|AAT02761.1| & AhpA, protection against organic peroxides & Emericella nidulans & 53.1 & $8 e-06$ \\
\hline ref|XP_659624.1| & hypothetical protein AN2020.2 & Aspergillus nidulans FGSC A4 & 53.1 & $8 e-06$ \\
\hline \multicolumn{5}{|l|}{ Vd21Q1-QUEVd3F/R } \\
\hline ref|XP_391592.1| & hypothetical protein FG11416.1 & Gibberella zeae $\mathrm{PH}-1$ & 75.5 & $2 \mathrm{e}-12$ \\
\hline ref|XP_389742.1| & hypothetical protein FG09566.1 & Gibberella zeae $\mathrm{PH}-1$ & 68.9 & $1 e-10$ \\
\hline ref|XP_388085.1| & hypothetical protein FG07909.1 & Gibberella zeae $\mathrm{PH}-1$ & 67.8 & $3 e-10$ \\
\hline ref|XP_961794.1| & hypothetical protein NCU05257 & Neurospora crassa OR74A & 62.8 & $1 e-08$ \\
\hline ref|XP_001932443.1] & conserved hypothetical protein & Pyrenophora tritici-repentis Pt-1C-BFP & 62.0 & $2 e-08$ \\
\hline ref|XP_389223.1| & hypothetical protein FG09047.1 & Gibberella zeae PH-1 & 59.3 & $1 e-07$ \\
\hline ref|XP_001800433.1| & hypothetical protein SNOG_10151 & Phaeosphaeria nodorum SN15 & 57.0 & $6 e-07$ \\
\hline ref|XP_001223987.1| & hypothetical protein CHGG_04773 & Chaetomium globosum CBS 148.51 & 57.0 & $6 e-07$ \\
\hline ref|XP_363804.2| & hypothetical protein MGG_01730 & Magnaporthe grisea 70-15 & 55.8 & $1 e-06$ \\
\hline ref|XP_001555211.1| & hypothetical protein BC1G_06341 & Botryotinia fuckeliana B05.10 & 38.1 & 0.27 \\
\hline ref|XP_001596875.1| & hypothetical protein SS1G_03098 & Sclerotinia sclerotiorum 1980 & 37.4 & 0.45 \\
\hline \multicolumn{5}{|l|}{ Vd21Q1-FLAQ2 } \\
\hline ref|XP_384886.1 & hypothetical protein FG04710.1 & Gibberella zeae PH-1 & 114 & $4 e-24$ \\
\hline ref|XP_001402423.1| & hypothetical protein An10g00300 & Aspergillus niger CBS 513.88 Penicillium & 76.3 & $9 e-13$ \\
\hline emb|CAP98741.1| & Pc22g14530, secondary transporter & chrysogenum Wisconsin 54-1255 & 75.5 & $2 e-12$ \\
\hline ref|XP_001382383.1| & maltose permease & Pichia stipitis CBS 6054 & 70.5 & $5 e-11$ \\
\hline ref|XP_722051.1| & potential maltose permease & Candida albicans SC5314 & 70.5 & $5 e-11$ \\
\hline dbj|BAG11496.1| & alpha-glucoside permease & Saccharomyces cerevisiae & 69.7 & $8 e-11$ \\
\hline emb|CAX41832.1| & alpha-glucoside transporter, putative & Candida dubliniensis CD36 & 68.9 & $1 e-10$ \\
\hline ref|XP_001385456.1| & maltose permease & Pichia stipitis CBS 6054 & 68.2 & $2 e-10$ \\
\hline ref|XP_001526533.1| & hypothetical protein LELG_01361 & Lodderomyces elongisporus NRRL YB-4239 & 67.0 & $5 e-10$ \\
\hline ref|XP 001262269.1| & MFS maltose permease, putative & Neosartorya fischeri NRRL 181 & 67.0 & $5 e-10$ \\
\hline$\overline{\text { ref|NP_011805.1| }}$ & Maltose permease, inducible high-affinity & Saccharomyces cerevisiae & 66.6 & $7 e-10$ \\
\hline ref|XP_747524.1| & MFS maltose permease & Aspergillus fumigatus Af293 Ajellomyces & 65.5 & $2 e-09$ \\
\hline $\mathrm{gb|EEH07499.1|}$ & trehalose transporter & capsulatus G186AR & 65.1 & $2 e-09$ \\
\hline emb|CAB46745.1| & maltose permease & Kluyveromyces lactis & 64.7 & $3 e-09$ \\
\hline$\overline{g b|E E D 13883.1|}$ & MFS maltose permease, putative & Talaromyces stipitatus ATCC 10500 & 63.9 & $5 e-09$ \\
\hline dbj|BAG11506.1 & alpha-glucoside permease & Saccharomyces cerevisiae & 63.9 & $5 e-09$ \\
\hline gb|EED14705.1| & sugar transporter, putative & Talaromyces stipitatus ATCC 10500 & 63.5 & $6 e-09$ \\
\hline gb|EED14397.1| & sugar transporter, putative & Talaromyces stipitatus ATCC 10500 & 62.8 & $1 e-08$ \\
\hline gb|EDP49077.1| & MFS sugar transporter, putative & Aspergillus fumigatus A1163 & 62.8 & $1 e-08$ \\
\hline ref|XP_572882.1 & sugar transporter & Cryptococcus neoformans JEC21 & 62.0 & $2 e-08$ \\
\hline gb|EED24629.1| & hexose carrier protein, putative & Talaromyces stipitatus ATCC 10500 & 61.6 & $2 e-08$ \\
\hline ref|XP_568347.1 & trehalose transport-related protein & Cryptococcus neoformans JEC21 & 60.8 & $4 e-08$ \\
\hline gb|EED53463.1| & sugar transporter, putative & Aspergillus flavus NRRL3357 Saccharomyces & 60.5 & $5 e-08$ \\
\hline $\mathrm{gb}|\mathrm{ABV} 21349.1|$ & maltose transporter & pastorianus & 60.5 & $5 e-08$ \\
\hline ref|XP_001261420.1| & maltose permease & Neosartorya fischeri NRRL 181 Cryptococcus & 60.5 & $5 e-08$ \\
\hline ref|XP_571538.1| & trehalose transport-related protein & neoformans JEC21 & 60.5 & $5 e-08$ \\
\hline emb|CAD36557.1 & maltotriose symporter & Saccharomyces pastorianus & 60.5 & $5 e-08$ \\
\hline$\overline{\text { ref|XP_571470.1| }}$ & alpha-glucoside transport-related protein & Cryptococcus neoformans JEC21 & 60.5 & $5 e-08$ \\
\hline ref|XP_001258307.1| & maltose permease & Neosartorya fischeri NRRL 181 & 59.3 & $1 e-07$ \\
\hline
\end{tabular}




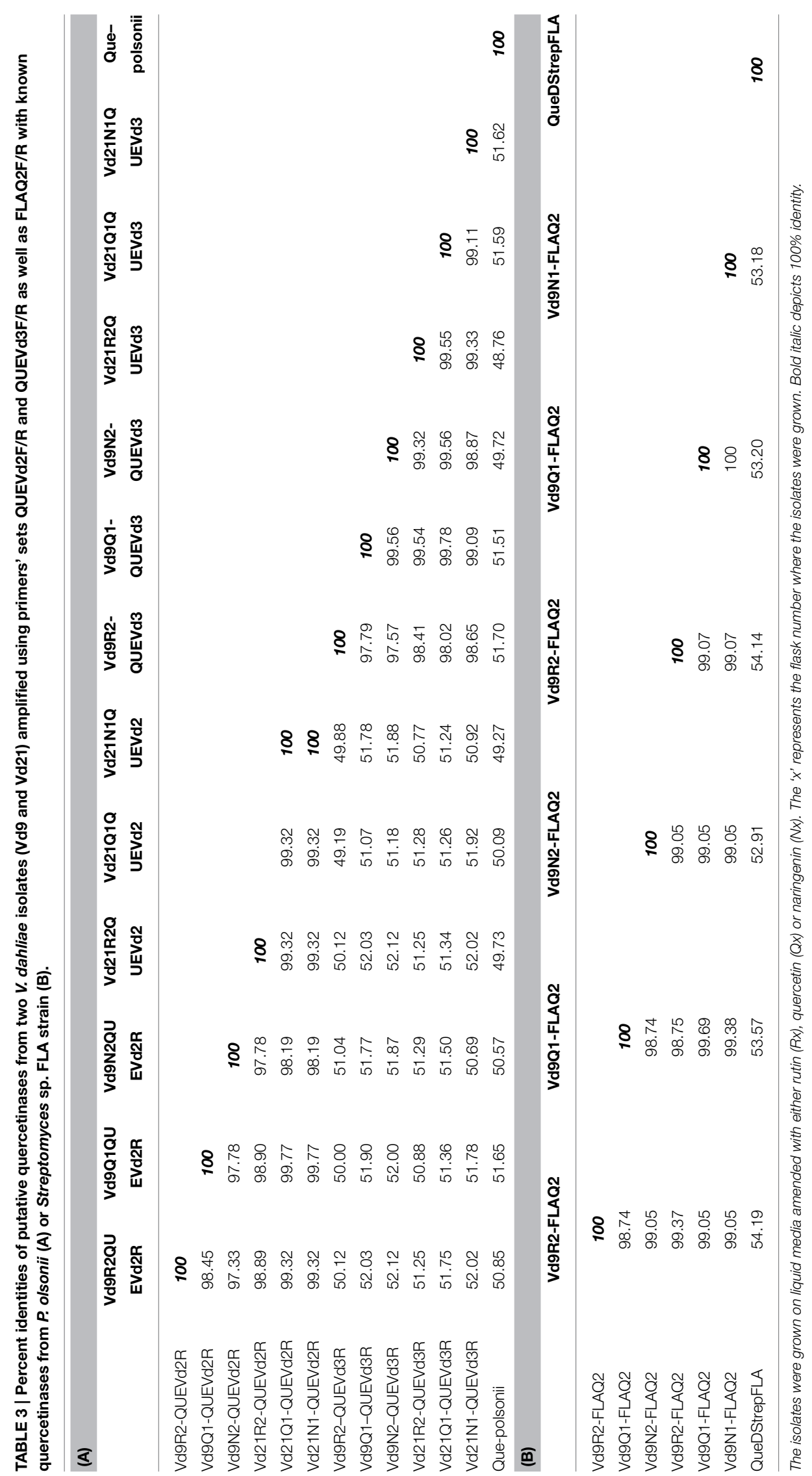




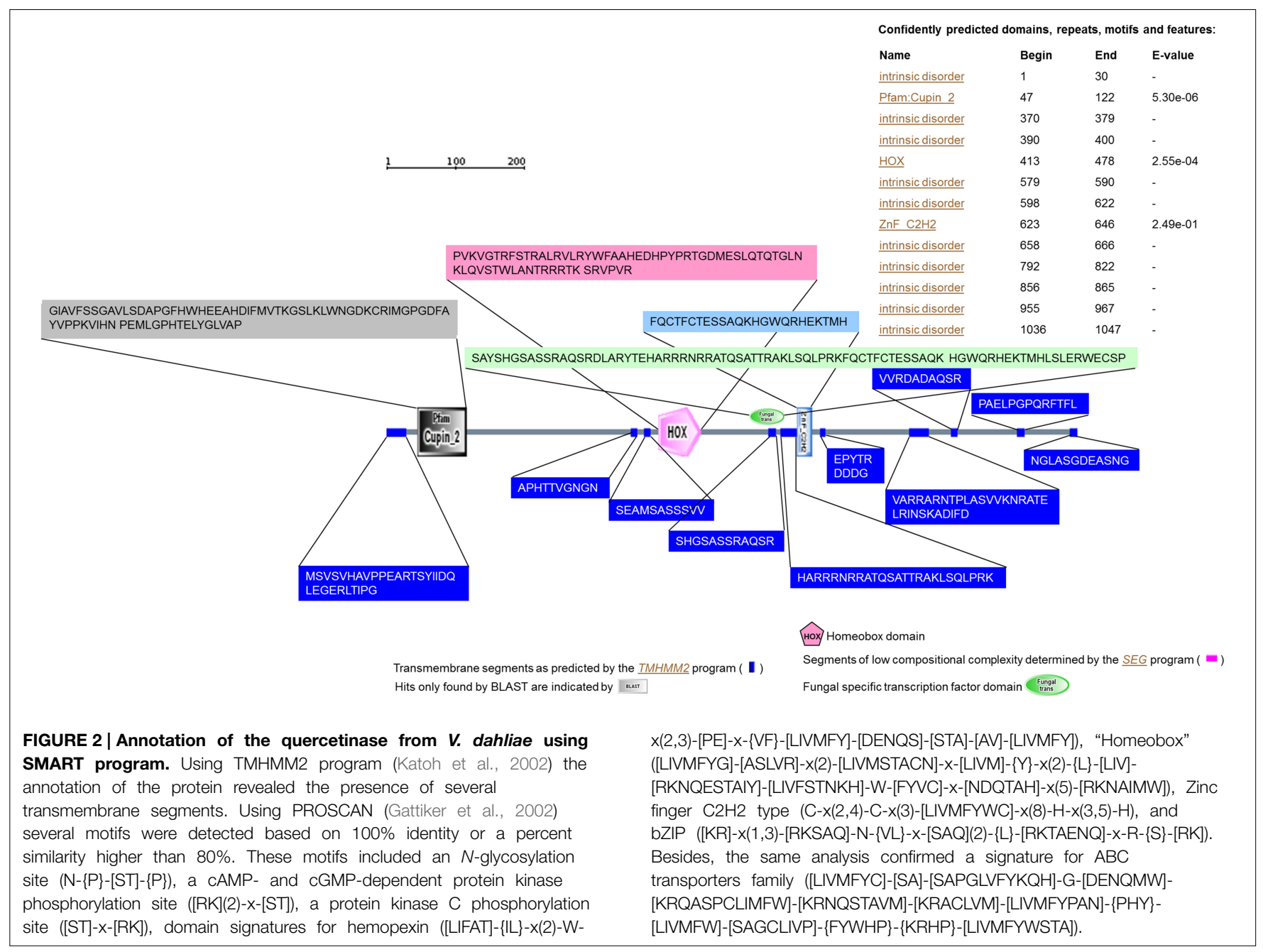

quercetinase was similar among the two tested isolates $\mathrm{Vd} 9$ and $\mathrm{Vd} 21$ (Figure 4C).

\section{Analyses of the Mutagenesis of VdQase Gene}

VdQase gene replacement mutant was produced by ATMT of the mutagenized gene into isolate Vd9. Sequence analyses of the mutagenized plasmids (pDHtVdQaseTn25) confirmed the insertion of EZ::TN after the 355th base of the second intron of the VdQase coding sequence. PCR analysis confirmed the replacement of the wild type copy of VdQase gene in the mutant transformants (Figure 5A). Southern blot analyses confirmed the insertion of a single copy of the hygromycin marker gene (Figure 5B). Two of the analyzed transformants (Figure 5C), Vd9+25-5 (M1), Vd9+25-7 (M2), were selected for further analyses based on their morphology, growth rate and spore production that were similar to the wild type.

\section{VdQase is Involved in Pathogenicity}

The findings of the present study along with our previous findings clearly indicated the ability of $V$. dahliae to metabolize the flavonol compound rutin due to its glucosidases and rhamnosidases which cleave the sugar moieties of this flavonol compound, thereby freeing the quercetin aglycone. The extracellularly-secreted quercetinases would break down quercetin and release a bi-product protocatechuic acid (PA). It was hypothesized that $V$. dahliae quercetinases may be involved in the pathogenicity of this soil-borne pathogen through utilization of the flavonol compound rutin. To test this hypothesis, we constructed a knock-out insertional mutant. The results revealed that VdQase mutants display reduced pathogenicity in potatoes as compared to the wild type isolate Vd9 (Figure 6). While the wild-type isolate produced severe chlorosis and wilting symptoms on the infected plants, the mutants produced very few wilt symptoms during the entire period of the assay. Interestingly, the VdQase mutants were more aggressive than weakly aggressive isolate Vs06-14.

Both AUDPC \% infection and severity were lower with the VdQase mutants than with the wild type aggressive isolate (Vd9), whereas the mutants had higher aggressiveness compared to the weakly aggressive isolate (Vs06-14). Stunting and vascular discoloration symptoms caused by the mutants were less pronounced than in response to either wild type highly aggressive isolate Vd9 or weakly aggressive isolate Vs06-14. 

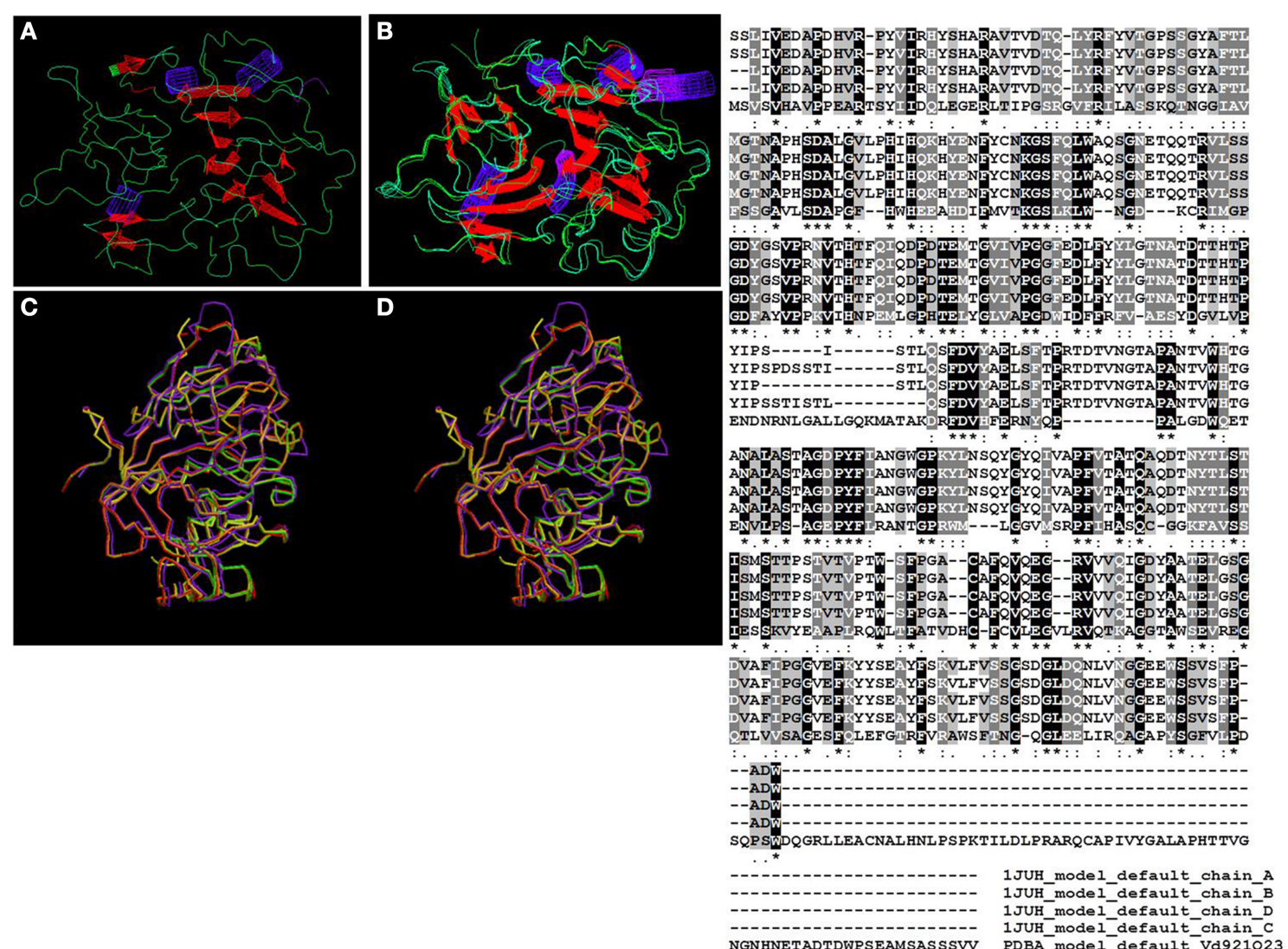

FIGURE 3 | Theoretical model generated using Whatlf program illustrating the structure of the quercetinase from $V$. dahliae (A) and its superposition with the template quercetin 2,3-dioxygenase from A. japonicus (B). (C, D) are outputs of the SuperPose program, where (D) represents the $V$. dahliae protein and (C) the the template quercetin 2,3-dioxygenase from $A$. japonicus. Minor conformational changes can be noticed between the two structures. The ClustalW2 alignment conducted by the SuperPose program during the superposition of the quercetinase protein from $V$. dahliae (PDBA_Vd921Q23) and the template quercetin 2,3-dioxygenase from $A$. japonicus (1JUH_), which have four chains labeled (A-D) highlights the high degree of conservation between the two structures. "*" means that the residues in the column are identical in all aligned sequences; ":" means that conserved substitutions have been observed; "." means that semi-conserved substitutions are observed; "-" means that there is a gap between the aligned sequences.

\section{Role of VdQase in Rutin and Quercetin Utilization}

To confirm our hypotheses regarding the utilization of the flavonol compound rutin and its break down product quercetin by $V$. dahliae, we first aimed to assess the flavonol compounds qualitatively using fluoresecence microscopy with the potato stem sections inoculated with wild type or VdQase mutants. The use of fluorescence microscopy to assay rutin utilization in planta revealed brighter yellow florescence in the tissues infected with the VdQase mutants as compared to the wild type and the non-inoculated control (Figure 7A). The fluorescence intensity in response to the mutant was similar to the weakly aggressive isolate Vs06-14 (Figure 7A). These results primarily suggest the involvement of VdQase in metabolism of the flavonol compound rutin. To unravel the facts of rutin utilization by $V$. dahliae by the extracellularly secreted quercetinases, the wild type and the mutant strains were grown in CDX medium supplemented with rutin and quercetin. A lower percentage of non-utilized rutin was recorded in presence of the highly aggressive wild type as compared to the
VdQase mutants and the weakly aggressive wild type in planta (Figure 7B).

\section{Role of the VdQase in the Manipulation of the Host SA- and JA-Signaling Pathways}

To address the mechanisms how $V$. dahliae overcome the host defenses to regulate its pathogenicity, we quantified the PA along with the end products SA and JA in the tissues infected with the wild type and the mutant strains. Protocatechuic acid, a by-product of rutin catabolism, had higher levels in tissues infected with the highly-aggressive wild type isolate Vd9 and the VdQase mutants as compared to the weaklyaggressive wild type isolate Vs06-14 or the untreated control (Figure 8A). Levels of free and bound SA were higher in tissues infected with the wild type highly-aggressive isolate $\mathrm{Vd} 9$ as compared to the VdQase mutants and the weakly-aggressive wild type Vs06-14 (Figure 8B). Levels of free and bound SA were also two to three times higher in tissues inoculated with the highly aggressive wild type isolate Vd-9 as compared to the 

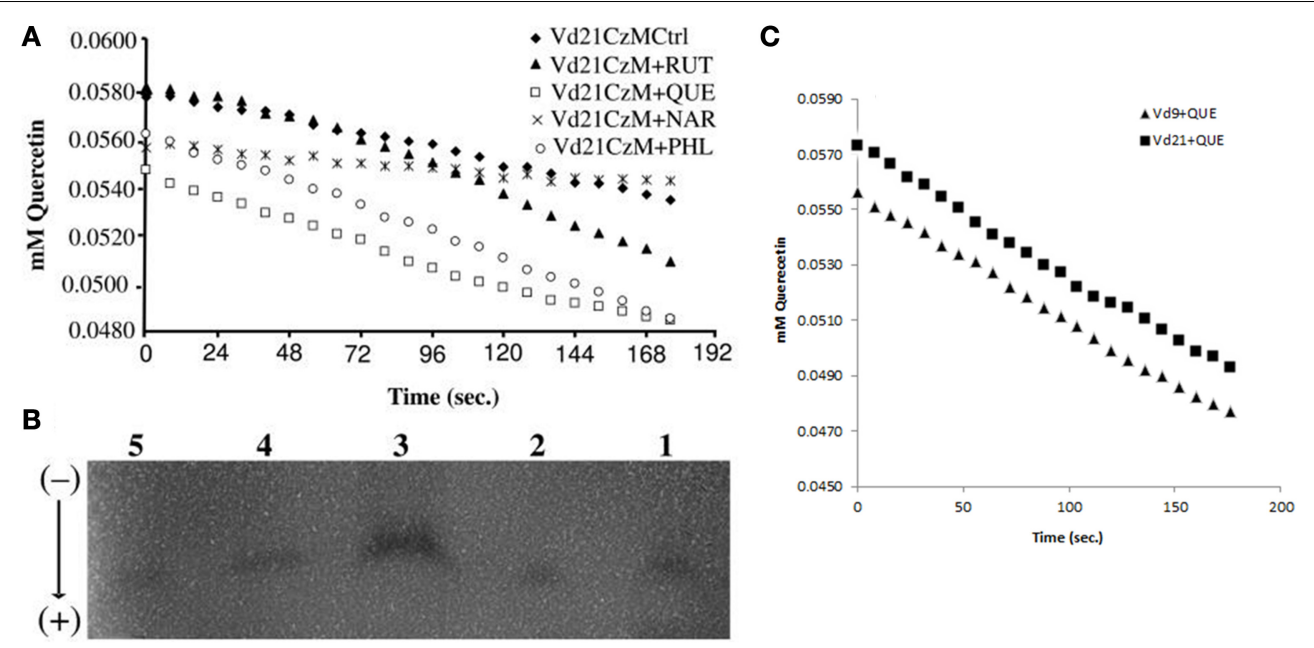

FIGURE 4 | (A) Quercetinase activity form V. dahliae isolate Vd21 grown on Czapeck-Dox liquid media (CzM) control (Ctrl, 1) or amended with rutin (RUT, 2), quercetin (QUE, 3), naringenin (NAR, 4), or phloroglucinol (PHL, 5), (B) isoforms detected on horizontal cellulose acetate electrophoresis system (Helena Laboratories, Beaumont, TX) and (C) comparison of the activity of the VdQase in the two tested isolates $\mathrm{Vd} 9$ and $\mathrm{Vd} 21$.
VdQase mutants (Figure 8B). However, SA levels in response to the VdQase mutants were higher than those in response to the weakly aggressive wild type Vs06-14. Quantification of JA revealed higher levels in potato leaves inoculated with the weakly aggressive isolate Vs06-14 as compared to highly aggressive wild type Vd9 (Figure 8C). JA levels in response to the VdQase mutants were lower than both the weakly- and highly-aggressive wild type isolates.

\section{Discussion}

In the current study, we have characterized a cupin domaincontaining protein with a quercetinase activity (VdQase) that seems to regulate $V$. dahliae's pathogenicity and contribute to its ability to counteract host defenses. Three primer sets designed in the conserved regions of known quercetinases from P. olsonii, A. japonicus and Streptomyces sp. strain FLA were used to amplify putative quercetinase-coding sequences in $V$. dahliae. Sequenced products were BLAST-searched against multiple databases and an ORF was located in the annotated $V$. dahliae genome available on MIT website (www.broadmit.edu/). Full-length transcripts were then isolated from mycelia incubated in presence of rutin or quercetin as well as other related flavonones (naringenin). A series of in silico analyses was conducted using amplified and sequenced $V$. dahliae products from the conserved region or the full length to determine various properties of each sequence. An in vitro analysis of the enzymatic activity followed to establish its role in cleaving quercetin. Furthermore, a mutagenesis approach using gene replacement in a highly-aggressive isolate $\mathrm{Vd} 9$ was used to establish the role of this gene in pathogenicity.

The $V$. dahliae sequences amplified using primer sets designed in the conserved regions of known quercetinases $(\mathrm{QueVd} 2 \mathrm{~F} / \mathrm{R}$, QueVd3F/R) showed significant hits with copper binding domains from the quercetin 2,3-dioxygenase of A. japonicus (E-value ranging from $1.00 e^{-06}$ to 0.01 ). Against the annotated $V$. dahliae genome the sequences showed $85-100 \%$ identity with cupin 1, Zn finger $\mathrm{C} 2 \mathrm{H} 2$ transcripts fully sequenced and annotated with no assigned function located on chromosome 3 of the two $V$. dahliae and $V$. albo-atrum reference isolates in the database. The ORF was then named VdQase.

Quercetin 2,3-dioxygenase (Q2,3D) was shown to be the only dioxygenase that functions as a homodimer and unequivocally depend on two moles of $\mathrm{Cu}^{2+}$ (Grotewold, 2006; Merkens et al., 2007; El Hadrami et al., 2009, 2011). Our investigation of the enzymatic activity of the purified protein showed a dependency on copper as a metal co-factor. Knowing that the mixture flavonol- $\mathrm{Cu}^{2+}$ can cause damage to fungal DNA in absence of reducing agents (Ahmed et al., 1994; Phillips, 1993), it is apparent that $V$. dahliae's quercetinase plays an important role in countering the fungitoxic secondary metabolites produced by the host plant (i.e., rutin, quercetin). The action of this enzyme is not restricted to quercetin since it was found to act on several related flavonols. However, the $K m$ and Vmax of this enzyme seem to depend on the $\mathrm{OH}$ topology at the $\mathrm{A}$ and $\mathrm{B}$ rings (Oka et al., 1972). In the present study, we showed the ability of the same enzyme to cleave other related flavonones (i.e., naringenin).

Sequences amplified using Q2FLAVd2F/R primers set showed, on the other hand, similarities with membrane permeases, sugar transporters/permeases, structural and transmembrane proteins, strongly suggesting a role as structural transmembrane proteins (cupin) with multiple functions, among which dioxygenation of flavnols/ones, i.e., querecetin and naringenin. The latter reaction seems to be favored by an acidification of the plasma membrane and the activity of an ATPase, as well as sugars binding/carrier motifs (i.e., ABC transporter, maltose permeases). This resembles what was described in animal and human cells in presence of a leukemia virus where sugar, quercetin and other flavonones were passively transported through the same transporters (Cunningham et al., 2006). It also agrees with other findings supporting a closer relationship between pathogenicity-related genes and those involved in the primary metabolism in Dothideomycetes i.e., 


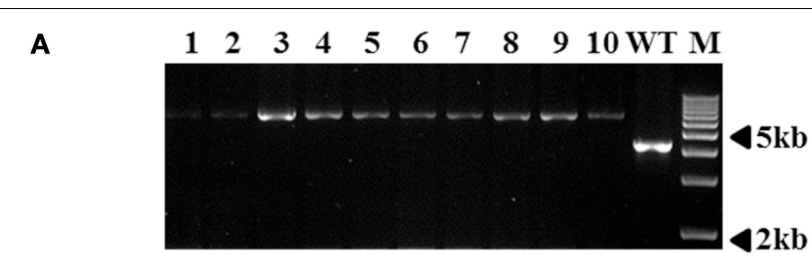

B

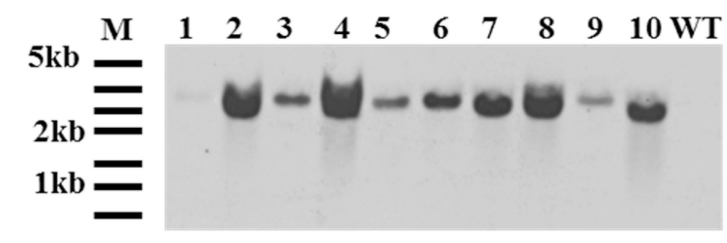

C

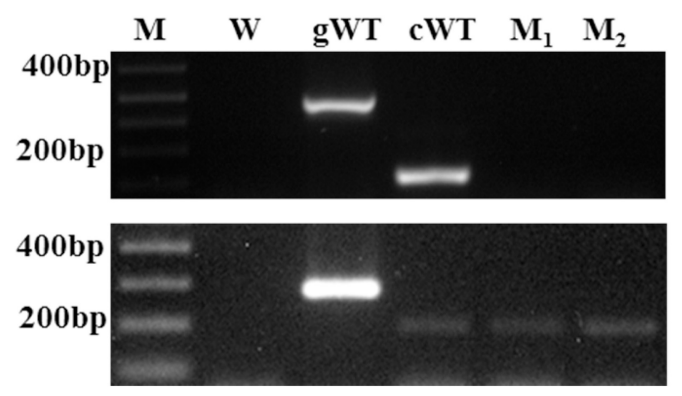

FIGURE 5 | Confirmation of VdQase mutant transformants by PCR, southern hybridization and RT-PCR analyses. (A) PCR confirmation of the double homologous recombination in the VdQase mutant by the replacement of wild type copy with mutant allele using a primer designed based on few hundred bp upstream sequence from the start codon and a VdQase gene specific reverse primer. M-Marker, WT-Wild type, Mutants (1-10). (B) Southern hybridization analyses of the wild type and the mutants with $E$ coRl digested genomic DNA and hygromycin B as a probe. M-Marker, WT-Wild type, Mutants (1-10). (C) RT-PCR analyses. M-Marker, W-Water control, gWT-Genomic DNA wild type, cWT-cDNA wild type, M1-Vd9+25-5 (Mutant 1) and M2-Vd9+25-7 (Mutant 2). The lower panel shows the expression of Actin in the wild type (both genomic and cDNA) and the VdQase mutant strains (M1 and M2).

Stagnospora nodurum and Leptosphaeria maculans (Rahman et al., 1989; Schoefer et al., 2003; Simpson et al., 1960).

Full-length transcripts of VdQase(s) were isolated from mycelia incubated in presence of rutin or quercetin as well as other related flavonones (naringenin). Two homolog genes 1.5 and $2.2 \mathrm{~Kb}$ in size were amplified using primer sets $\mathrm{Vd} 23 \mathrm{~F} / \mathrm{R} 1$ and FLAVd2F/R1, respectively, suggesting the presence of multiple quercetinases in $V$. dahliae. The analysis of these transcripts revealed a strong homology and 24-26\% identity with RmlC-like Cupin from A. japonicus or B. subtilis; both known as Quercetin 2,3-dioxygenase-like proteins. A series of in silico annotations and analysis including the generation of a PDB signature of the predicted proteins revealed with certainty matches (E-value $<$ 1.00e-06) with quercetin 2,3-dioxygenases from A. japonicus and B. subtilis. In addition, the in vitro analysis of the enzymatic activity confirmed its role in cleaving quercetin and established the characteristics of this enzyme. This confirms earlier data showing the detection of the by-product of detoxification of quercetin by UPLC-MS/MS (El Hadrami et al., 2011).

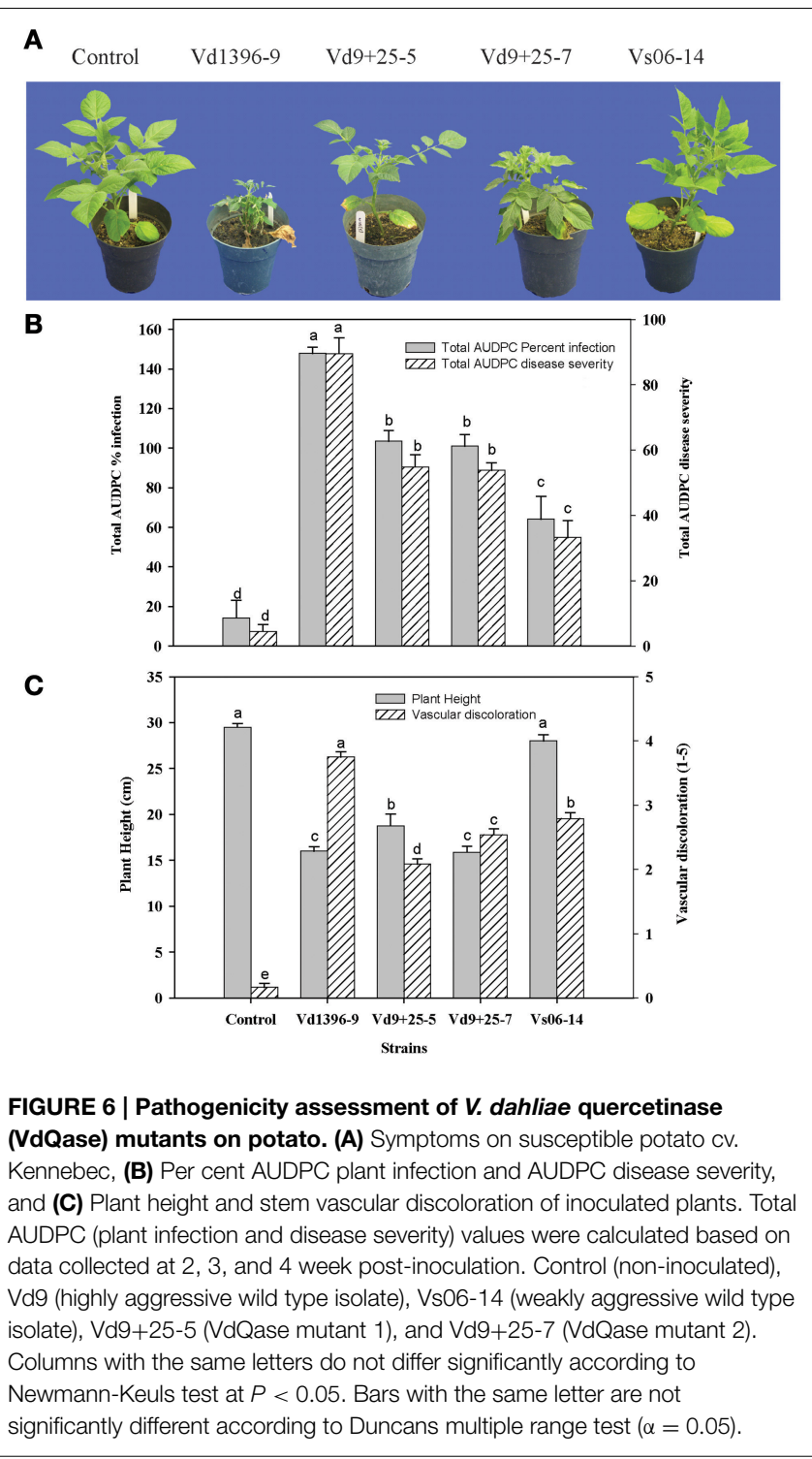

To tentatively establish a role of VdQase in $V$. dahliae pathogenicity, a gene replacement mutagenesis approach was used in a highly-aggressive isolate Vd9. Mutants with single copies of the hygromycin marker gene were successfully generated. Two of them that did not show any impairment in morphology, growth rate or spore production as compared to the wild type were further used to inoculate potato plants and assess disease progress as well as accumulation of rutin in planta. The VdQase mutants exhibited an attenuated pathogenicity as compared to their counterpart wild type and highly aggressive isolate $\mathrm{Vd}$ 9, suggesting that VdQase is a pathogenicity-related gene that plays a key role in the expression of Verticillium wilt. However, disease severity caused by these mutants was higher than that of the wild type and weakly aggressive isolate Vs06-14. This suggests either (i) an absence of VdQase in Vs06-14 or (ii) the amount of rutin induced in planta upon infection with Vs0614 is not enough to induce the expression of the VdQase. An alternative hypothesis would be related to the fact that multiple 

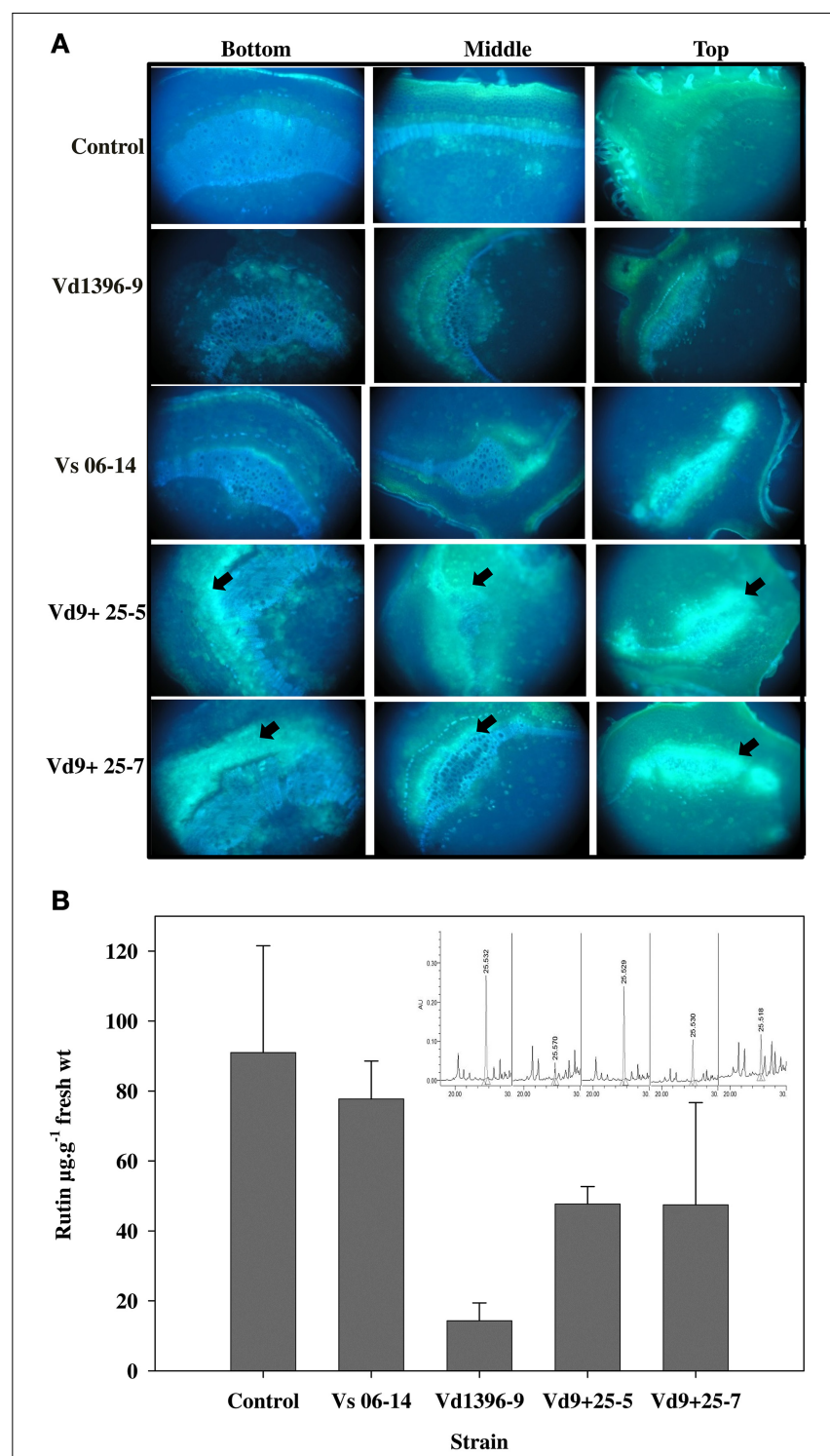

FIGURE 7 | Utilization of flavonol compounds by $V$. dahliae quercetinase (VdQase) mutants in planta. (A) Fluorescence microscopy showing the accumulation of the flavonol compounds in the potato stem infected with $V$. dahliae wild type and the mutants at $28 \mathrm{dpi}$ as indicated by the yellow fluorescence detected by immersing the cross sectioned potato stem in $1 \%$ Neu's reagent. (B) In planta utilization of rutin by VdQase mutant as quantified by HPLC. HPLC chromatograms were shown at the top right corner for rutin. Control (non-inoculated), Vd9 (highly aggressive wild type), Vs06-14 (a weakly aggressive wild type), Vd9+25-5 (VdQase mutant 1), and Vd9+25-7 (VdQase mutant 2). Arrows indicate the accumulation of flavonoids including rutin and quercetin in potato tissues.

putative VdQases were detectable in $V$. dahliae, allowing the generated mutants to have an intermediate phenotype and to be more aggressive than the weakly-aggressive and wild type isolate Vs06-14.

Knowing from an earlier study (El Hadrami et al., 2011) that rutin is the main secondary metabolites induced in potato to a high level $(>100 \mu \mathrm{M})$ by successful bicontrol agents and only weakly by the non-effective agents, and having characterized

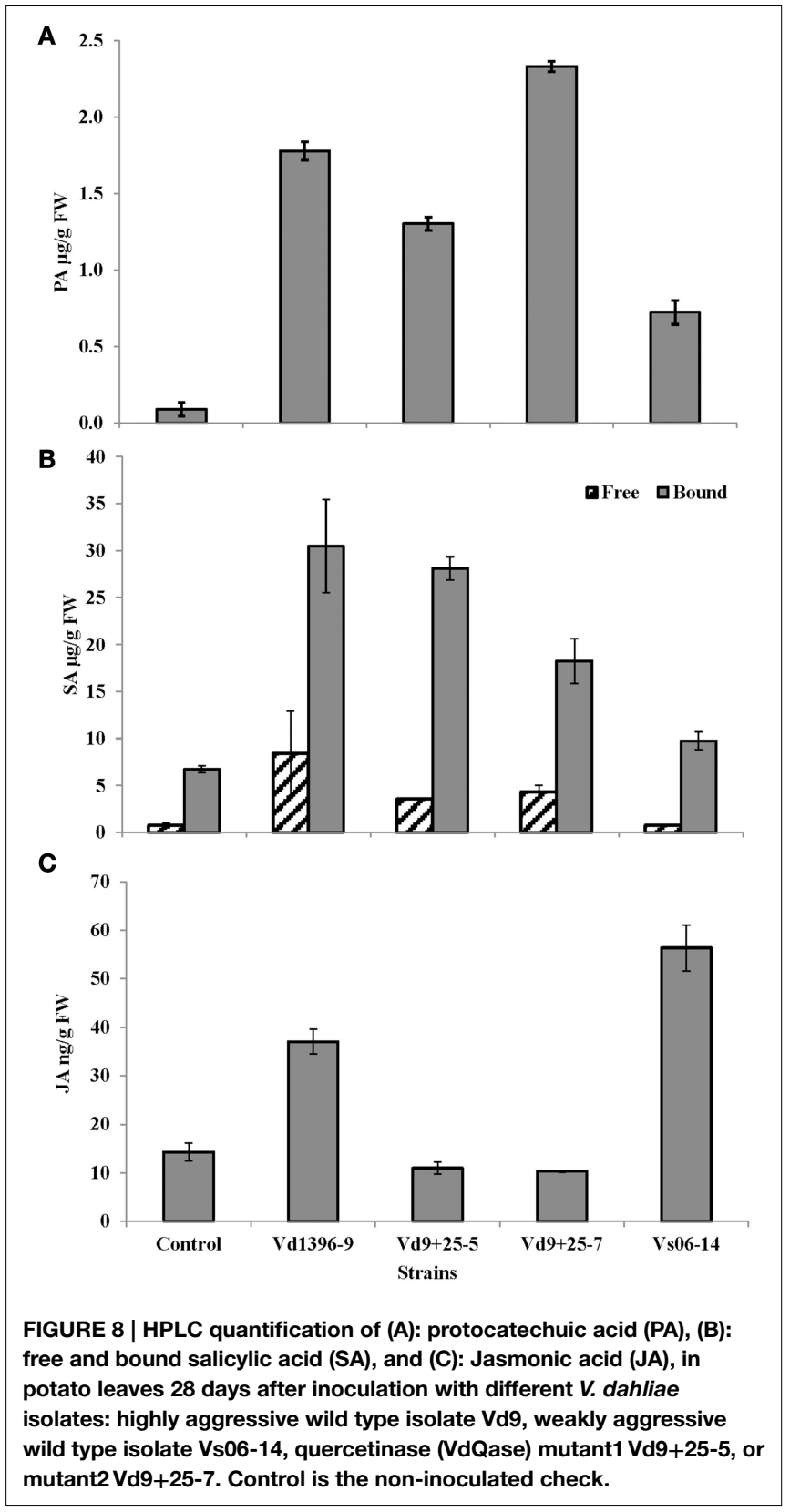

in the present study $V$. dahliae' enzyme responsible for its catabolism, it appears that the success of $V$. dahliae in the rhizosphere is partly enhanced by its ability to exhibit a quercetinase activity that allows it to counter host plants' defenses (i.e., synthesis/accumulation of flavonols/ones), turning them into by-products such as 2-PCPGCA (Supplementary Material 1). The latter may play a role as a defensive or an offensive effector in $V$. dahliae counter-defenses/pathogenicity either directly or indirectly. The phloroglucinol moiety as an antibiotic, may, when released, create disequilibrium among the microbial communities including pathogens and antagonists, thereby helping $V$. dahliae's notoriety in the rhizosphere. The protocatechuic part can be converted to benzoate and salicylates, which can induce SA-related defenses in planta (Figure 9). This 


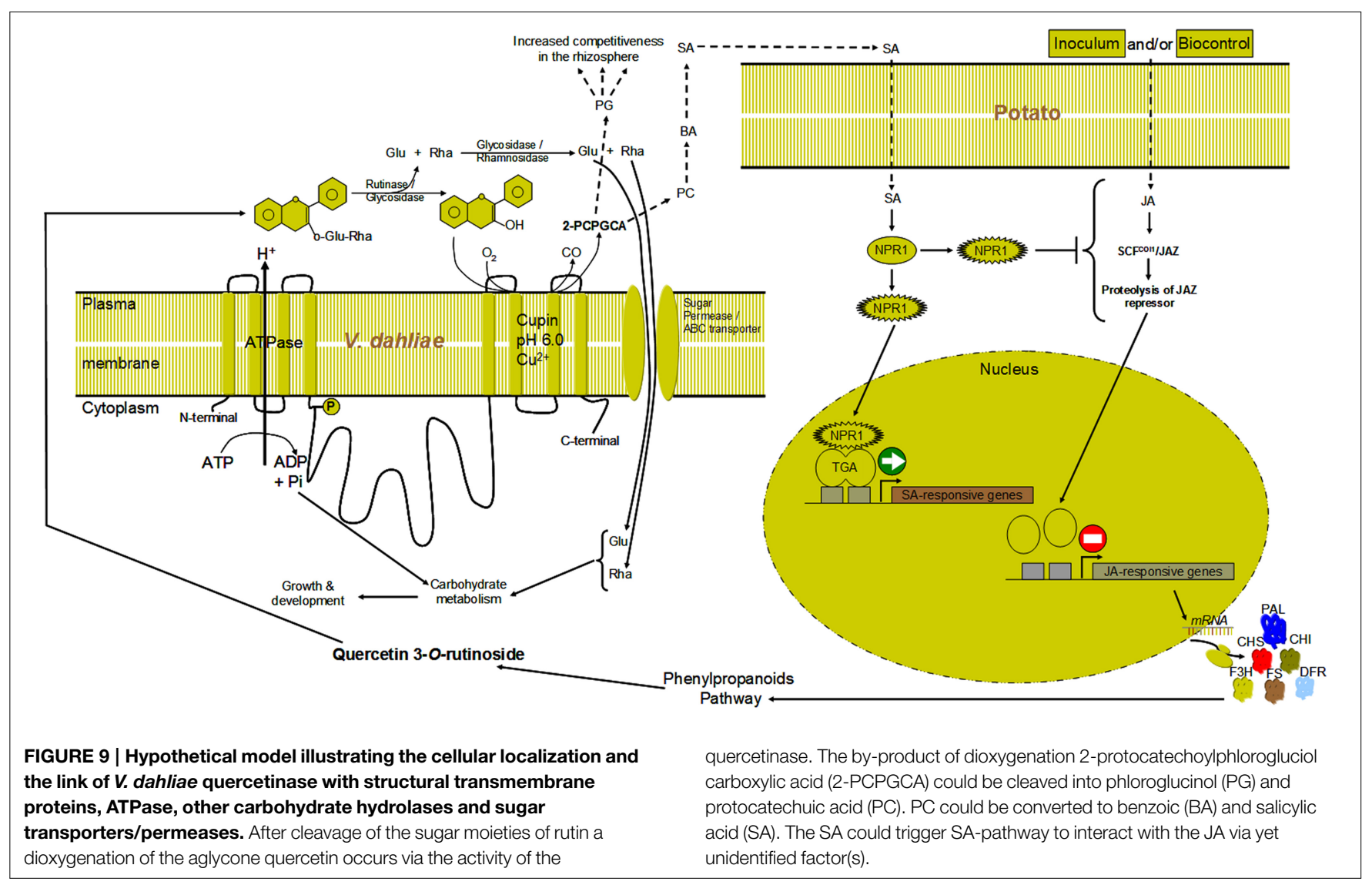

may interfere indirectly with JA-related potato-defenses against $V$. dahliae, in line with the negative feedback between SA and JA pathways (Derksen et al., 2013). The quantification of PA, SA, JA in planta revealed higher levels of PA and SA in response to highly-aggressive and wild type isolate $\mathrm{Vd} 9$ as well as the mutants as compared to the control of the weakly-aggressive and wild type isolate Vs06-14. Oppositely, JA content were higher in response to weakly-aggressive and wild type isolate Vs06-14 as compared to highly-aggressive and wild type isolate $\mathrm{Vd} 9$ and the mutants. This supports our data from an earlier study showing that Canada milkvetch produces jasmonate-related compounds and mediated JA-related defenses in infected potato plants (El Hadrami et al., 2011).

VdQase mutants exhibited lower pathogenicity than the highly aggressive wild type isolate $\mathrm{Vd} 9$ and a lower utilization of rutin and quercetin both in vitro and in planta, suggesting an involvement of this enzyme in key steps of the plant x pathogen interactions. That is the catabolic detoxification of quercetin through dioxygenation. Detection of the protocatechuic acid and phloroglucinol, end products of rutin/quercetin catabolism, confirmed the activity of this enzyme. Assessment of SA and JA revealed lower inductions in response to the VdQase mutants and the weakly aggressive wild type isolate Vs06-14 as compared to the wild type highly aggressive isolate $\mathrm{Vd} 9$. This confirms our earlier report on the action of rutin in a dose response manner. Higher JA triggers higher synthesis and accumulation of rutin $(>100 \mu \mathrm{M})$, which is detoxified by the VdQase of highly aggressive wild type isolate. The consequence of such a detoxification is the elicitation of SA pathway (higher free SA). VdQase mutants induce lower levels of JA, leading to a low synthesis and accumulation of rutin, which is either not enough to induce the VdQase activity or generate only low levels of SA to interfere with the JA pathway. Elicitation of one pathway (i.e., SA-pathway) to hijack another pathway that is more adequate for defense (i.e., JA-pathway) had similarly been described in tobacco in response to Pseudomonas syringae (Rayapuram and Baldwin, 2007), A. thaliana in response to F. oxysporum (Thatcher et al., 2009) and tomato in response to Botrytis (El Oirdi et al., 2011).

In the present study we were able to characterize in silico and in vitro the VdQase enzyme responsible for the catabolism of the main secondary metabolite induced in potato plant (rutin) in response to infection by $V$. dahliae or elicitation by biocontrol agents. We also generated mutants to functionally confirm the role of this enzyme. Based on an indication that rutin can be transported systemically in planta (Buer et al., 2007), a zigzag race (Figure 9) between the pathogen and the host, would lead either to an enhanced host defense if the rutin accumulates at levels high enough to restrict the pathogen's growth and/or development, or to a counter-defense by the pathogen in case this flavonol glycoside accumulates below a certain threshold level. Biocontrol agents to reduce Verticillium wilt in potato could be efficient if they are able to induce this metabolite at higher level but, in the light of the present findings, their application could be managed to alter the zigzag scheme during the interaction. 


\section{Author Contributions}

All co-authors contributed to the conception of the ideas, design of the experiments, analysis, and data interpretation of different parts of the study. FD supervised the work. AE, $\mathrm{MI}$, and LA run the pathogenicity tests. AE completed the gene expression, in-silico annotation, structure and identification. MI generated the VQase mutants and run the functional analysis tests. AE and LA did the chromatography analyses for the identification and quantification of metabolites. AE, $\mathrm{MI}$, and FD wrote the manuscript, and all co-authors reviewed it.

\section{References}

Ahmed, M. S., Ramesh, V., Nagaraja, V., Parish, J. H., and Hadi, S. M. (1994). Mode of binding of quercetin to DNA. Mutagenesis 9, 193. doi: 10.1093/mutage/9.3.193

Alkher, H., El Hadrami, A., Rashid, K. Y., Adam, L. R., and Daayf, F. (2009). Crosspathogenicity of Vertcillium dahliae between potato and sunflower. Eur. J. Plant Pathol. 124, 505-519. doi: 10.1007/s10658-009-9437-Z

Altschul, S. F., Madden, T. L., Schaffer, A. A., Zhang, Z., Miller, W., and Lipman, D. J. (1997). Gapped BLAST and PSI-BLAST: a new generation of protein database search programs. Nucleic Acids Res. 25, 3389-3402. doi: 10.1093/nar/25. 17.3389

Andersen, Ø. M., and Markham, K. R. (2006). “Preface," in Flavonoids: Chemistry, Biochemistry and Applications, eds Ø. M. Andersen and K. R. Markham (Boca Raton, FL: CRC Press; Taylor and Francis Group).

Barry, K. M., Davies, N. W., and Mohammed, C. L. (2002). Effect of season and different fungi on phenolics in response to xylem wounding and inoculation in Eucalyptus nitens. For. Pathol. 32, 163-178. doi: 10.1046/j.14390329.2002.00281.x

Bartz, W. (1971). Über den abbau aromatischer verbindungen durch Fusarium oxysporum Schlecht. Arch. Mikrobiol. 78, 341-352. doi: 10.1007/BF00412274

Bates, P. A., Kelley, L. A., MacCallum, R. M., and Sternberg, M. J. (2001). Enhancement of protein modeling by human intervention in applying the automatic programs 3D-JIGSAW and 3D-PSSM. Proteins 5, 39-46. doi: $10.1002 /$ prot. 1168

Bendtsen, J. D., Nielsen, H., von Heijne, G., and Brunak, S. (2004). Improved prediction of signal peptides: Signalp 3.0. J. Mol. Biol. 340, 783-795. doi: 10.1016/j.jmb.2004.05.028

Bennet, R. N., and Wallsgrove, R. M. (1994). Secondary metabolites in plant defence mechanisms. New Phytol. 127, 617-633. doi: 10.1111/j.14698137.1994.tb02968.x

Braune, A., Gutschow, M., Engst, W., and Blaut, M. (2001). Degradation of quercetin and luteolin by Eubacterium ramulus. Appl. Environ. Microbiol. 67, 5558-5567. doi: 10.1128/AEM.67.12.5558-5567

Buer, C. S., Muday, G. K., and Djordjevic, M. A. (2007). Long distance transport flavonoids. Plant Physiol. 145, 478-490. doi: 10.1104/pp.107.101824

Cheng, J., Randall, A., Sweredoski, M., and Baldi, P. (2005). SCRATCH: a protein structure and structural feature prediction server. Nucleic Acids Res. 33, W72-W76. doi: 10.1093/nar/gki396

Child, J. J., Simpson, F. J., and Westlake, D. W. S. (1963). Degradation of rutin by Aspergillus flavus. Production, purification, and characterization of an esterase. Can. J. Microbiol. 9, 653-664. doi: 10.1139/m63-087

Cunningham, P., Afzal-Ahmed, I., and Naftalin, R. J. (2006). Docking studies show that D-glucose and quercetin slide through the transporter GLUT1. J. Biol. Chem. 281, 5797-5803. doi: 10.1074/jbc.M509422200

Daayf, F. (2015). Verticillium wilts in crop plants: pathogen invasion and host defense responses. Can. J. Plant Pathol. 37, 8-20. doi: 10.1080/07060661.2014.989908

Daayf, F., and Lattanzio, V. (eds.) (2008). Recent Advances in Polyphenol Research, Vol. 1. Oxford, UK: Blackwell Publishing Ltd.

\section{Acknowledgments}

This research was supported by a grant from the Natural Sciences and Engineering Research Council (NSERC-Canada) to Dr. Fouad Daayf. The authors are grateful to Omex Agriculture Inc. for its publication fees contribution.

\section{Supplementary Material}

The Supplementary Material for this article can be found online at: http://journal.frontiersin.org/article/10.3389/fpls.2015. 00440/abstract

Derksen, H., Rampitsch, C., and Daayf, F. (2013). Signaling cross-talk in plant disease resistance. Plant Sci. 207, 79-87. doi: 10.1016/j.plantsci.2013. 03.004

Dobinson, K. F., Grant, S. J., and Kang, S. (2004). Cloning and targeted disruption, via Agrobacterium tumefaciens-mediated transformation, of a trypsin protease gene from the vascular wilt fungus Verticillium dahliae. Curr. Genet. 45, 104-110. doi: 10.1007/s00294-003-0464-6

Dobinson, K. F., Lecomte, N., and Lazarovits, G. (1997). Production of an extracellular trypsin-like protease by the fungal plant pathogen Verticillium dahliae. Can. J. Microbiol. 43, 227-233. doi: 10.1139/w06-033

El Hadrami, A., Adam, L. R., and Daayf, F. (2011). Biocontrol treatments confer protection against Verticillium dahliae infection of potato by inducing anti-microbial metabolites. Mol. Plant Microbe Interact. 24, 328-335. doi: 10.1094/MPMI-04-10-0098

El Hadrami, A., El Hadrami, I., and Daayf, F. (2009). "Suppression of induced plant defense responses by fungal pathogens," in Molecular-Plant Microbe Interactions, Chap. 10, eds K. Bouarab, N. Brisson, and F. Daayf (CABI), 231-268. doi: 10.1079/9781845935740.0231

El Oirdi, M., Abd El Rahman, T., Rigano, L., El Hadrami, A., Rodriguez, M. C., Daayf, F., et al. (2011). Botrytis cinerea manipulates the antagonistic effects between immune pathways to promote disease development in tomato. Plant Cell 23, 2405-2421. doi: 10.1105/tpc.111.083394

Finn, R. D., Mistry, J., Schuster-Bockler, B., Griffiths-Jones, S., and Hollich, V., Lassmann, T., et al. (2006). Pfam: clans, web tools and services. Nucleic Acids Res. 34, D247-D251. doi: 10.1.1.104.3603

Fusetti, F., Schröter, K. H., Steiner, R. A., van Noort, P. I., Pijning, T., Rozeboom, H. J., et al. (2002). Crystal structure of the copper-containing quercetin 2,3-dioxygenase from Aspergillus japonicus. Structure 10, 259-268. doi: 10.1016/S0969-2126(02)00704-9

Gattiker, A., Gasteiger, E., and Bairoch, A. (2002). Scanprosite: a reference implementation of a prosite scanning tool. Appl. Bioinformatics 1, 107-108.

Gomi, M., Sonoyama, M., and Mitaku, S. (2004). High performance system for signal peptide prediction: Sosuisignal. Chem-Bio Informatics J. 4, 142-147. doi: $10.1273 /$ cbij. 4.142

Gotoh, O. (1999). Multiple sequence alignment: algorithms and applications. $A d v$. Biophys. 36, 159-206. doi: 1016/S0065-227X(99)80007-0

Grotewold, E. (2006). The Science of Flavonoids. New York, NY: Springer.

Harborne, J. B., and Williams, C. A. (2000). Advances in flavonoid research since 1992. Phytochemistry 55, 481-504. doi: 10.1016/S0031-9422(00)00235-1

Hund, H. K., Breuer, J., Lingens, F., Hüttermann, J., Kappl, R., and Fetzner, S. (1999). Flavonol 2,4-dioxygenase from Aspergillus niger DSM 821, a type 2 CuII-containing glycoprotein. Eur. J. Biochem. 263, 871-878. doi: 10.1046/j.1432-1327.1999.00574.x

Hurst, C. J. Crawford, R. L., Garland, J. L., Lipson, D. A., Mills, A. L., and Stetzenbach, L. D. (2002). Manual of Environmental Microbiology, 2nd Edn. Washington, DC: ASM Press. doi: 10.1128/9781555815882

Katoh, K., Misawa, K., Kuma, K., and Miyata, T. (2002). MAFFT: a novel method for rapid multiple sequence alignment based on fast Fourier transform. Nucleic Acids Res. 30, 3059-3066. doi: 10.1093/nar/gkf436 
Kelley, L. A., and Sternberg, M. J. E. (2009). Protein structure prediction on the web: a case study using the Phyre server. Nat. Protoc. 4, 363-371. doi: 10.1038/nprot.2009.2

Krogh, A., Larsson, B., von Heijne, G., and Sonnhammer, E. L. (2001). Predicting transmembrane protein topology with a hidden Markov model: application to complete genomes. J. Mol. Biol. 305, 567-580. doi: 10.1006/jmbi.2000.4315

Kunst, F., Ogasawara, N., and Moszer, I., et al. (1997). The complete genome sequence of the gram-positive bacterium Bacillus subtilis. Nature 390, 249-256. doi: $10.1038 / 36786$

Lagrange, H., Jay-Allgmand, C., and Lapeyrie, F. (2001). Rutin, the phenolglycoside from eucalyptus root exudates, stimulates Pisolithus hyphal growth at picomolar concentrations. New Phytol. 149, 349-355. doi: 10.1046/j.14698137.2001.00027.x

Larkin, M. A., Blackshields, G., Brown, N. P., Chenna, R., McGettigan, P. A., McWilliam, M., et al. (2007). Clustal W and Clustal X version 2.0. Bioinformatics 23, 2947-2948. doi: 10.1093/bioinformatics/btm404

Laskowski, R. A., Watson, J. D., and Thornton, J. M. (2005). Profunc: a server for predicting protein function from 3d structure. Nucleic Acids Res. 33, W89-W93. doi: 10.1093/nar/gki414

Letunic, I., Copley, R. R., Schmidt, S., Ciccarelli, F. D., Doerks, T., Schultz, J., et al. (2004). SMART 4.0: towards genomic data integration. Nucleic Acids Res. 32, D142-D144. doi: 10.1093/nar/gkh088

Maiti, R., Van Domselaar, G. H., Zhang, H., and Wishart, D. S. (2004). SuperPose: a simple server for sophisticated structural superposition. Nucleic Acids Res. 1, W590-W594. doi: 10.1093/nar/gkh477

Martin, F., Duplessis, S., Ditengou, F., Lagrange, H., Voiblet, C., and Lapeyrie, F. (2001). Development cross talking in the ectomycorrhizal symbiosis: signals and communication genes. New Phytol. 151, 145-154. Doi: 10.1046/j.14698137.2001.00169.x

Merkens, H., Sielker, S., Rose, K., and Fetzner, S. (2007). A new mocupin quercetinase of Streptomyces sp. FLA- identification and heterologous expression of the queD gene and activity of the recombinant enzyme towards different flavonols. Arch. Microbiol. 187, 475-487. doi: 10.1007/s00203-0070215-z

Meuwly, P., and Métraux, J. P. (1993). Ortho-anisic acid as internal standard for the simultaneous quantitation of salicylic acid and its putative biosynthetic precursors in cucumber leaves. Anal. Biochem. 214, 500-505. doi: 10.1006/abio.1993.1529

Mullins, E. D., Chen, X., Romaine, P., Raina, R., Geiser, D. M., and Kang, S. (2001). Agrobacterium tumefaciens-mediated transformation of Fusarium oxysporum: an efficient tool for insertional mutagenesis and gene transfer. Phytopathology 91, 173-180. doi: 10.1094/PHYTO.2001.91.2.173

Oka, T., and Simpson, F. J. (1971). Quercetinase, a dioxygenase containing copper. Biochem. Biophys. Res. Commun. 43, 1-5. Doi: 10.1073/pnas.262506299

Oka, T., Simpson, F. J., Child, J. J., and Mills, C. (1971). Degradation of rutin by Aspergillus flavus. Purification of the dioxygenase, querecentinase. Can. J. Microbiol. 17, 111-118. doi: 10.1139/m71-019

Oka, T., Simpson, F. J., and Krishnamurty, H. G. (1972). Degradation of rutin by Aspergillus Xavus. Studies on specificity, inhibition, and possible reaction mechanism of quercetinase. Can. J. Microbiol. 18, 493-508. doi: 10.1139/ $\mathrm{m} 72-076$

Peters, N. K., Frost, J. W., and Long, S. R. (1986). A plant flavone, luteoline, induces expression of Rhizobium melilotii nodulation genes. Science 233, 977-980. doi: 10.1126/science. 3738520

Phillips, D. A. (1993). "Flavonoids: plant signal to soil microbes," in Phenolic Metabolism in Plants. Recent Advances in Phytochemistry, Vol. 26, eds H. A. Stafford and P. K. Ibrahim (New York, NY: Plenum Press), 201-231. doi: 10.1007/978-1-4615-3430-3_7

Poulin, M. J., Bel-Rhlid, R., Piché, Y., and Chenevert, R. (1993). Flavonoids released by carrot (Daucus carrota) seedlings stimulate hyphal development of vesiculararbuscular mycorrhizal fungi in presence of optimal $\mathrm{CO}_{2}$ enrichment. J. Chem. Ecol. 19, 2317-2327. doi: 10.1007/BF00979666

Quevillon, E., Silventoinen, V., Pillai, S., Harte, N., Mulder, N., and Apweiler, R., et al. (2005). Interproscan: protein domains identifier. Nucleic Acids Res. 33, W116-W120. doi: 10.1.1.283.6201

Rahman, A., Shahabuddin, Hadi, S. M., Parish, J. H., and Ainley, K. (1989). Strand scission in DNA induced by quercetin and $\mathrm{Cu}(\mathrm{II})$ : role of $\mathrm{Cu}(\mathrm{I})$ and oxygen free radicals. Carcinogenesis 10, 1833-1839. doi: 10.1093/carcin/10. 10.1833

Ramachandran, G. N., Ramakrishnan, C., and Sasisekharan, V. (1963). Stereochemistry of polypeptide chain configurations. J. Mol. Biol. 7, 95-99. doi: 10.1016/S0022-2836(63)80023-6

Rayapuram, C., and Baldwin, I. T. (2007). Increased SA in NPR1-silenced plants antagonizes JA and JA-dependent direct and indirect defenses in herbivore-attacked Nicotiana attenuata in nature. Plant J. 52, 700-715. doi: 10.1111/j.1365-313X.2007.03267.x

Remy, E., Meyer, M., Blaise, F., Simon,U. K., Kuhn, D., Balesdent, M. H., et al. (2009). Key enzyme of the Leloir pathway is involved in pathogenicity of Leptosphaeria maculans toward oilseed rape. Mol. Plant Microbe Interact. 22, 725-736. doi: 10.1094/MPMI-22-6-0725

Rose, K., and Fetzner, S. (2006). Identification of linear plasmid pAM1 in the flavonoid degrading strain Actinoplanes missouriensis T (DSM 43046). Plasmid 55, 249-254. doi: 10.1016/j.plasmid.2005.10.003

Rozema, J., Bjorn, L. O., Bornman, J. F., Gaberscik, A., Hader, D. P., Trost, T., et al. (2002). The role of UV-B radiation in aquatic and terrestrial ecosystems - an experimental and functional analysis of the evolution of UVabsorbing compounds. J. Photochem. Photobiol. 66, 2-12. doi: 10.1016/S10111344(01)00269-X

Sariaslani, F. S., and Dalton, H. (1989). Microbial enzymes for oxidation of organic molecules. Crit. Rev. Biotechnol. 9, 171-257. doi: 10.3109/073885589090 36736

Schoefer, L., Mohan, R.,Schwiertz, A., Braune, A., and Blaut, M. (2003). Anaerobic degradation of flavonoids by Clostridium orbiscindens. Appl. Environ. Microbiol. 69, 5849-5854. doi: 10.1128/AEM.69.10.5849-5854.2003

Schwede, T., Kopp, J., Guex, N., and Peitsch, M. C. (2003). Swiss-model: an automated protein homology-modeling server. Nucleic Acids Res. 31, 3381-3385. doi: 10.1093/nar/gkg520

Simpson, F. J., Talbot, G., and Westlake, D. W. S. (1960). Production of carbon monoxide in the enzymatic degradation of rutin. Biochem. Biophys. Res. Comm. 2, 15-18. doi: 10.1016/0006-291X(60)90255-2

Steiner, R. A., Meyer-Klaucke, W., and Dijkstra, B. (2002). Functional analysis of the copper-dependent quercetin 2,3-dioxygenase. 2. X-ray absorption studies of native enzyme and anaerobic complexes with the substrates quercetin and myricetin. Biochemistry 41, 7963-7968. doi: 10.1021/ bi015974y

Thatcher, L. F., Manners, J. M., and Kazan, K. (2009). Fusarium oxysporum hijicks COI1-mediated jasmonate signaling in order to promote disease development in Arabidopsis. Plant J. 58, 927-939. doi: 10.1111/j.1365-313X.2009. 03831.x

Tranchimand, S., Ertel, G., Gaydou, V., Gaudin, C., Tron, T., and Iacazio, G. (2008). Biochemical and molecular characterization of a quercetinase from Penicillium olsonii. Biochimie 90, 781-789. doi: 10.1016/j.biochi. 2007.12.004

Treutter, D. (2006). Significance of flavonoids in plant resistance: a review. Environ. Chem. Lett. 4, 147-157. doi: 10.1007/s10311-006-0068-8

Uppal, A. K., El Hadrami, A., Adam, L. R., Tenuta, M., and Daayf, F. (2007). Pathogenic variability of Verticillium dahliae Kleb. isolates from potato fields in Manitoba and screening of bacteria for their biocontrol. Can. J. Plant Pathol. 29, 141-152. doi: 10.1080/0706066070 9507450

Uppal, A. K., El Hadrami, A., Adam, L. R., Tenuta, M., and Daayf, F. (2008). Biological control of potato Verticillium wilt under controlled and field conditions using selected bacterial antagonists and plant extracts. Biol. Control. 44, 90-100. doi: 10.1016/j.biocontrol.2007.10.020

Viornery, L., Saliba, C., Daskiewicz, J. B., Bayet, C., Comte, G., Fenet, B., et al. (2000). Phenylpropanoids from Umbilicus pendulinus. Chem. Pharm. Bull. 48, 1768-1770. doi: $10.1248 / \mathrm{cpb} .48 .1768$

Vriend, G. (1990). WHAT IF: a molecular modeling and drug design program. J. Mol. Graph. 8, 52-56. doi: 10.1016/0263-7855(90)80070-V

Wass, M. N., and Sternberg, M. J. (2008). ConFunc-functional annotation in the twilight zone. Bioinformatics 24, 798-806. doi: 10.1093/bioinformatics/ btn037

Westlake, D. W. S., Roxburgh, J. M., and Talbot, G. (1961). Microbial production of carbon monoxide from flavonoids. Nature 189, 510-511. doi: 10.1038/189510a0 
Winter, J., Moore, L. H., Dowell Jr, V. R., and Bokkenheuser, V. D. (1989). C-ring cleavage of flavonoids by human intestinal bacteria. Appl. Environ. Microbiol. 55, 1203-1208.

Winter, J., Popoff, M. R., Grimont, P., and Bokkenheuser, V. D. (1991). Clostridium orbiscindens sp. nov., a human intestinal bacterium capable of cleaving the flavonoid C-ring. Int. J. Syst. Bacteriol. 41, 355-357. doi: 10.1099/00207713-413-355

Wray, J. W., and Abeles, R. H. (1993). A bacterial enzyme that catalyzes formation of carbon monoxide. J. Biol. Chem. 268, 21466-21469.
Conflict of Interest Statement: The authors declare that the research was conducted in the absence of any commercial or financial relationships that could be construed as a potential conflict of interest.

Copyright (C) 2015 El Hadrami, Islam, Adam and Daayf. This is an open-access article distributed under the terms of the Creative Commons Attribution License (CC BY). The use, distribution or reproduction in other forums is permitted, provided the original author(s) or licensor are credited and that the original publication in this journal is cited, in accordance with accepted academic practice. No use, distribution or reproduction is permitted which does not comply with these terms. 